\title{
Restricting supercharacters of the finite group of unipotent uppertriangular matrices
}

\author{
Nathaniel Thiem* \\ Department of Mathematics \\ University of Colorado at Boulder \\ thiemn@colorado.edu
}

\author{
Vidya Venkateswaran ${ }^{\dagger}$ \\ Department of Mathematics \\ California Institute of Technology \\ vidyav@caltech.edu
}

Submitted: Aug 22, 2008; Accepted: Feb 9, 2009; Published: Feb 20, 2009

Mathematics Subject Classification: 05E99, 20C33

\begin{abstract}
It is well-known that understanding the representation theory of the finite group of unipotent upper-triangular matrices $U_{n}$ over a finite field is a wild problem. By instead considering approximately irreducible representations (supercharacters), one obtains a rich combinatorial theory analogous to that of the symmetric group, where we replace partition combinatorics with set-partitions. This paper studies the supercharacter theory of a family of subgroups that interpolate between $U_{n-1}$ and $U_{n}$. We supply several combinatorial indexing sets for the supercharacters, supercharacter formulas for these indexing sets, and a combinatorial rule for restricting supercharacters from one group to another. A consequence of this analysis is a Pieri-like restriction rule from $U_{n}$ to $U_{n-1}$ that can be described on set-partitions (analogous to the corresponding symmetric group rule on partitions).
\end{abstract}

\section{Introduction}

The representation theory of the finite group of upper-triangular matrices $U_{n}$ is a wellknown wild problem. Therefore, it came as somewhat of a surprise when C. André was able to show that by merely "clumping" together some of the conjugacy classes and some of the irreducible representations one attains a workable approximation to the representation theory of $U_{n}[1,2,3,4]$. In his Ph.D. thesis [14], N. Yan showed how the algebraic geometry of the original construction could be replaced by more elementary constructions. E. AriasCastro, P. Diaconis, and R. Stanley [8] were then able to demonstrate that this theory can in fact be used to study random walks on $U_{n}$ using techniques that traditionally required

${ }^{*}$ The authors would like to thank Diaconis and Marberg for many enlightening discussions regarding this work, and anonymous referees for their comments.

†Part of this work is Venkateswaran's honors thesis at Stanford University. 
knowledge of the full character theory [11]. Thus, the approximation is fine enough to be useful, but coarse enough to be computable.

André's approximate theory also has a remarkable combinatorial structure that recalls the classical connection between the representation theory of the symmetric group and partition combinatorics. In this case, we replace partition with set-partitions, so that

$$
\left\{\begin{array}{c}
\text { Almost irreducible } \\
\text { representations of } U_{n}
\end{array}\right\} \stackrel{1-1}{\longleftrightarrow}\left\{\begin{array}{c}
\text { Set partitions } \\
\text { of }\{1,2, \ldots, n\}
\end{array}\right\} .
$$

In particular, the number of almost irreducible representations is a Bell number (or more generally a $q$-analogue of a Bell number). One of the main results of this paper is to extend the analogy with the symmetric group by giving a combinatorial Pieri-like formula for set-partitions that corresponds to restriction in $U_{n}$.

Our strategy is to study a family of groups - called pattern groups - that interpolate between $U_{n}$ and $U_{n-1}$. A pattern group is a unipotent matrix group associated to a poset $\mathcal{P}$ of $\{1,2, \ldots, n\}$ subject to the condition that the $(i, j)$ th can be nonzero only if $i \preceq j$ in $\mathcal{P}$ (a group version of the incidence algebra of $\mathcal{P}$ ). For example, $U_{n}$ is the pattern group associated to the poset $1 \prec 2 \prec \cdots \prec n$, and our interpolating pattern groups are associated to the posets $2 \prec 3 \prec \cdots \prec n$ and $1 \prec m$ for some $1<m \leq n$.

In [10], P. Diaconis and M. Isaacs generalized André's theory to the notion of a supercharacter theory for arbitrary finite groups, where irreducible characters are replaced by supercharacters and conjugacy classes are replaced by superclasses. In particular, their paper generalized André's original construction by giving a supercharacter theory for pattern groups (and even more generally algebra groups). The combinatorics of these supercharacter theories for general pattern groups is not yet understood: there seems to be a constant tension between the set partition combinatorics of $U_{n}$ and the underlying poset $\mathcal{P}$ (see, for example, [12]). In particular, lengthy anti-chains seem to imply more complicated combinatorics. Another main result of this paper is to work out the combinatorics for the set of interpolating subgroups, demonstrating that while for these posets the combinatorics becomes more technical, it remains computable.

In [10], Diaconis and Isaacs also showed that the restriction of a supercharacter between pattern groups is a $\mathbb{Z}_{\geq 0}$-linear combination of supercharacters in the subgroup. However, even for $U_{m} \subseteq U_{n}$, these coefficients are not well understood (and also depend on the particular embedding of $U_{m}$ in $U_{n}$ ). This paper offers a first step in understanding this problem giving an algorithm for computing coefficients. In general, these will be polynomials in the size $q$ of the underlying finite field, but it is unknown what these coefficients might count.

Section 2 reviews the basics of supercharacter theory and pattern groups. Section 3 defines the interpolating subgroups $U_{(m)}$, and finds two different sets of natural superclass and supercharacter representatives, which we call comb representatives and path representatives. Section 4 uses a general character formula from [12] to determine character formulas for both comb and path representatives. The character formula for comb representatives - Theorem 4.1 - is easier to compute directly, but the path representative character formula - Theorem 4.3 - has a more pleasing combinatorial structure. Section 
5 uses the character formulas to derive a restriction rule for the interpolating subgroups given in Theorem 5.1. Corollary 5.1 iterates these restrictions to deduce a recursive decomposition formula for the restriction from $U_{n}$ to $U_{n-1}$.

This paper is the companion paper to [13], which studies the superinduction of supercharacters. Other work related to supercharacter theory of unipotent groups, include C. André and A. Neto's exploration of supercharacter theories for unipotent groups of Lie types $B, C$, and $D[5]$, C. André and A. Nicolás' analysis of supertheories over other rings [6], and an intriguing possible connection between supercharacter theories and Boyarchenko and Drinfeld's work on $L$-packets [9].

\section{Preliminaries}

This section reviews several topics fundamental to our main results: Supercharacter theories, pattern groups, and a character formula for pattern groups.

\section{$2.1 \quad$ Supertheories}

Let $G$ be a group. As defined in [10], a supercharacter theory for $G$ is a partition $\mathcal{S}^{\vee}$ of the elements of $G$ and a set of characters $\mathcal{S}$, such that

(a) $|\mathcal{S}|=\left|\mathcal{S}^{\vee}\right|$

(b) Each $S \in \mathcal{S}^{\vee}$ is a union of conjugacy classes,

(c) For each irreducible character $\gamma$ of $G$, there exists a unique $\chi \in \mathcal{S}$ such that

$$
\langle\gamma, \chi\rangle>0
$$

where $\langle$,$\rangle is the usual innerproduct on class functions,$

(d) Every $\chi \in \mathcal{S}$ is constant on the elements of $\mathcal{S}^{\vee}$.

We call $\mathcal{S}^{\vee}$ the set of superclasses and $\mathcal{S}$ the set of supercharacters. Note that every group has two trivial supercharacter theories - the usual character theory and the supercharacter theory with $\mathcal{S}^{\vee}=\{\{1\}, G \backslash\{1\}\}$ and $\mathcal{S}=\left\{\mathbb{1}, \gamma_{G}-\mathbb{1}\right\}$, where $\mathbb{1}$ is the trivial character of $G$ and $\gamma_{G}$ is the regular character.

There are many ways to construct supercharacter theories, but this paper will study a particular version developed in [10] to generalize André's original construction to a larger family of groups called algebra groups.

\subsection{Pattern groups}

While many results can be stated in the generality of algebra groups, frequently statements become simpler if we restrict our attention to a subfamily called pattern groups. We follow the construction of [10] for the superclasses and supercharacters of pattern groups. 
Let $U_{n}$ denote the set of $n \times n$ unipotent upper-triangular matrices with entries in the finite field $\mathbb{F}_{q}$ of $q$ elements. For any poset $\mathcal{P}$ on the set $\{1,2, \ldots, n\}$, the pattern group $U_{\mathcal{P}}$ is given by

$$
U_{\mathcal{P}}=\left\{u \in U_{n} \mid u_{i j} \neq 0 \text { implies } i \leq j \text { in } \mathcal{P}\right\} .
$$

This family of groups includes unipotent radicals of rational parabolic subgroups of the finite general linear groups $\mathrm{GL}_{n}\left(\mathbb{F}_{q}\right)$; the group $U_{n}$ is the pattern group corresponding to the total order $1<2<3<\cdots<n$.

The group $U_{\mathcal{P}}$ acts on the $\mathbb{F}_{q}$-algebra

$$
\mathfrak{n}_{\mathcal{P}}=\left\{u-1 \mid u \in U_{\mathcal{P}}\right\}
$$

by left and right multiplication. Two elements $u, v \in U_{\mathcal{P}}$ are in the same superclass if $u-1$ and $v-1$ are in the same two-sided orbit of $\mathfrak{n}_{\mathcal{P}}$. Note that since every element of $U_{\mathcal{P}}$ can be decomposed as a product of elementary matrices, every element in the orbit containing $v-1 \in \mathfrak{n}_{\mathcal{P}}$ can be obtained by applying a sequence of the following row and column operations.

(a) A scalar multiple of row $j$ may be added to row $i$ if $j>i$ in $\mathcal{P}$,

(b) A scalar multiple of column $k$ may be added to column $l$ if $k<l$ in $\mathcal{P}$.

There are also left and right actions of $U_{\mathcal{P}}$ on the dual space $\mathfrak{n}_{\mathcal{P}}^{*}=\operatorname{Hom}_{\mathbb{F}_{q}}\left(\mathfrak{n}_{\mathcal{P}}, \mathbb{F}_{q}\right)$ given by

$$
(u \lambda v)(x-1)=\lambda\left(u^{-1}(x-1) v^{-1}\right), \quad \text { where } \lambda \in \mathfrak{n}_{\mathcal{P}}^{*}, u, v, x \in U_{\mathcal{P}}
$$

Fix a nontrivial group homomorphism $\theta: \mathbb{F}_{q}^{+} \rightarrow \mathbb{C}^{\times}$. The supercharacter $\chi^{\lambda}$ with representative $\lambda \in \mathfrak{n}_{\mathcal{P}}^{*}$ is

$$
\chi^{\lambda}=\frac{\left|U_{\mathcal{P}} \lambda\right|}{\left|U_{\mathcal{P}} \lambda U_{\mathcal{P}}\right|} \sum_{\mu \in U_{\mathcal{P}} \lambda U_{\mathcal{P}}} \theta \circ(-\mu) .
$$

We identify the functions $\lambda \in \mathfrak{n}_{\mathcal{P}}^{*}$ with matrices by the vector space isomorphism,

$$
\begin{aligned}
{[\cdot]: \mathfrak{n}_{\mathcal{P}}^{*} } & \longrightarrow \\
\lambda & \mapsto[\lambda]=\sum_{i<j \in \mathcal{P}} \lambda_{i j}\left(e_{i j}+\mathfrak{n}_{\mathcal{P}}^{\perp}\right),
\end{aligned}
$$

where $e_{i j} \in \mathfrak{n}_{\mathcal{P}}$ has $(i, j)$ entry 1 and zeroes elsewhere, $\lambda_{i j}=\lambda\left(e_{i j}\right)$, and

$$
\begin{aligned}
M_{n}\left(\mathbb{F}_{q}\right) & =\left\{n \times n \text { matrices with entries in } \mathbb{F}_{q}\right\}, \\
\mathfrak{n}_{\mathcal{P}}^{\perp} & =\left\{y \in M_{n}\left(\mathbb{F}_{q}\right) \mid y_{i j}=0 \text { for all } i<j \text { in } \mathcal{P}\right\} .
\end{aligned}
$$

We will typically choose the quotient representative to be in $\mathfrak{n}_{\mathcal{P}}$. Then, as with superclasses, every element in the orbit containing $\lambda \in \mathfrak{n}_{\mathcal{P}}^{*}$ can be obtained by applying a sequence of the following row and column operations to $[\lambda]$. 
(a) A scalar multiple of row $i$ may be added to row $j$ if $i<j$ in $\mathcal{P}$,

(b) A scalar multiple of column $l$ may be added to column $k$ if $l>k$ in $\mathcal{P}$.

Note that since we are in the quotient space $M_{n}\left(\mathbb{F}_{q}\right) / \mathfrak{n}_{\mathcal{P}}^{\perp}$, we quotient by all nonzero entries that might occur through these operations that are not in allowable in $\mathfrak{n}_{\mathcal{P}}$.

Example. For $U_{n}$ we have

$$
\left\{\begin{array}{c}
\text { Superclasses } \\
\text { of } U_{n}
\end{array}\right\} \longleftrightarrow\left\{\begin{array}{l|l}
u \in U_{n} & \begin{array}{l}
u-1 \text { has at most one nonzero } \\
\text { entry in every row and column }
\end{array}
\end{array}\right\}
$$

If $q=2$, then

$$
\left\{\begin{array}{l|l}
u \in U_{n} \mid \begin{array}{l}
u-1 \text { has at most one nonzero } \\
\text { entry in every row and column }
\end{array}
\end{array}\right\} \longleftrightarrow\left\{\begin{array}{c}
\text { Set partitions } \\
\text { of }\{1,2, \ldots, n\}
\end{array}\right\} .
$$

Similarly, if

$$
\mathfrak{n}_{n}=U_{n}-1
$$

then

$$
\left\{\begin{array}{c|c}
\begin{array}{c}
\text { Supercharacters } \\
\text { of } U_{n}
\end{array}
\end{array}\right\} \longleftrightarrow\left\{\lambda \in \mathfrak{n}_{n}^{*} \begin{array}{l}
\text { The matrix }[\lambda] \text { has at most one non- } \\
\text { zero entry in every row and column }
\end{array}\right\} .
$$

Let

$$
\mathcal{S}_{n}(q)=\left\{\lambda \in \mathfrak{n}_{n}^{*} \mid[\lambda] \text { has at most one nonzero entry in every row and column }\right\}
$$

\subsection{A supercharacter formula for pattern groups}

Let $U_{\mathcal{P}}$ be a pattern group with corresponding nilpotent algebra $\mathfrak{n}_{\mathcal{P}}$. Let

$$
J=\{(i, j) \mid i<j \text { in } \mathcal{P}\}
$$

Given $u \in U_{\mathcal{P}}$ and $\lambda \in \mathfrak{n}_{\mathcal{P}}^{*}$, define $a, b \in \mathbb{F}_{q}^{|J|}$ by

$$
\begin{array}{rlrl}
a_{i j} & =\sum_{j<k \text { in } \mathcal{P}} u_{j k} \lambda_{i k}, & & \text { for }(i, j) \in J, \\
b_{j k} & =\sum_{i<j \text { in } \mathcal{P}} u_{i j} \lambda_{i k}, & \text { for }(j, k) \in J .
\end{array}
$$

Let $M$ be the $|J| \times|J|$ matrix given by

$$
M_{i j, k l}=\left\{\begin{array}{ll}
u_{j k} \lambda_{i l}, & \text { if } i<j<k<l \text { in } \mathcal{P}, \\
0, & \text { otherwise. }
\end{array} \quad \text { for }(i, j),(k, l) \in J .\right.
$$


Informally, if one superimposes the matrices $u$ and $[\lambda]$, then

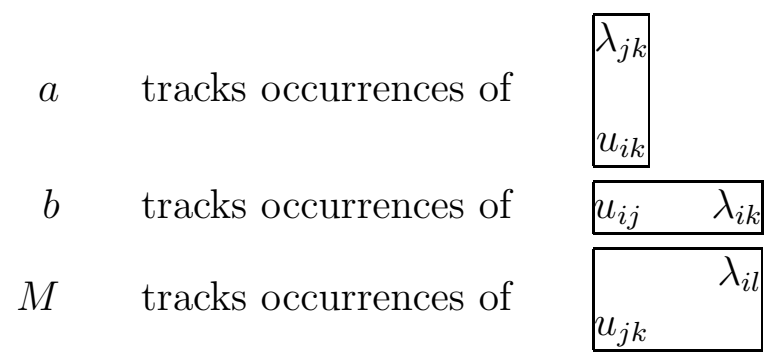

Remark. Each of $a, b$, and $M$ depend on $u, \lambda$, and $\mathcal{P}$. However, to make the notation less heavy-handed, we leave this dependence out of the notation.

Let $\operatorname{Null}(M)$ denote the nullspace of $M$ and let $:: \mathbb{F}_{q}^{|J|} \times \mathbb{F}_{q}^{|J|} \rightarrow \mathbb{F}_{q}$ be the usual inner product (dot product) on $\mathbb{F}_{q}^{|J|}$. The following theorem gives a general supercharacter formula for pattern groups. However, typical applications of the theorem make a particular choice of superclass and supercharacter representatives.

Theorem 2.1 ([12]). Let $u \in U_{\mathcal{P}}$ and $\lambda \in \mathfrak{n}_{\mathcal{P}}^{*}$. Then

(a) The character

$$
\chi^{\lambda}(u)=0
$$

unless there exists $x \in \mathbb{F}_{q}^{|J|}$ such that $M x=-a$ and $b \cdot \operatorname{Null}(M)=0$,

(b) If $\chi^{\lambda}(u)$ is not zero, then

$$
\chi^{\lambda}(u)=\frac{q^{\left|U_{\mathcal{P}} \lambda\right|}}{q^{\operatorname{rank}(M)}} \theta(x \cdot b) \theta \circ \lambda(u-1),
$$

where $x \in \mathbb{F}_{q}^{|J|}$ is such that $M x=-a$.

Remark. There are two natural choices for $\chi^{\lambda}$, one of which is the conjugate of the other. Theorem 2.1 uses the convention of [10] rather than [12].

C. André proved the $U_{n}$-version of this supercharacter formula for large characteristic [3], and [8] extended it to all finite fields. Note that the following theorem follows from Theorem 2.1 by choosing appropriate representatives for the superclasses and supercharacters.

Theorem 2.2. Let $\lambda \in \mathcal{S}_{n}(q)$, and let $u \in U_{n}$ be a superclass representative as in (2). Then

(a) The character degree

$$
\chi^{\lambda}(1)=\prod_{i<j, \lambda_{i j} \neq 0} q^{j-i-1}
$$


(b) The character

$$
\chi^{\lambda}(u)=0
$$

unless whenever $u_{j k} \neq 0$ with $j<k$, we have $\lambda_{i k}=0$ for all $i<j$ and $\lambda_{j l}=0$ for all $l>k$.

(c) If $\chi^{\lambda}(u) \neq 0$, then

$$
\chi^{\lambda}(u)=\frac{\chi^{\lambda}(1) \theta \circ \lambda(u-1)}{q^{\left|\left\{i<j<k<l \mid u_{j k}, \lambda_{i l} \in \mathbb{F}_{q}^{\times}\right\}\right|}} .
$$

\section{Interpolating between $U_{n-1}$ and $U_{n}$}

Fix $n \geq 1$. For $2 \leq m \leq n$, let

$$
\begin{aligned}
U_{(m)} & =\left\{u \in U_{n} \mid u_{1 j}=0, \text { for } 1<j \leq m\right\}=U_{\mathcal{P}_{(m)}}, \\
\mathfrak{n}_{(m)} & =\left\{u-1 \mid u \in U_{(m)}\right\}=\mathfrak{n}_{\mathcal{P}_{(m)}},
\end{aligned}
$$

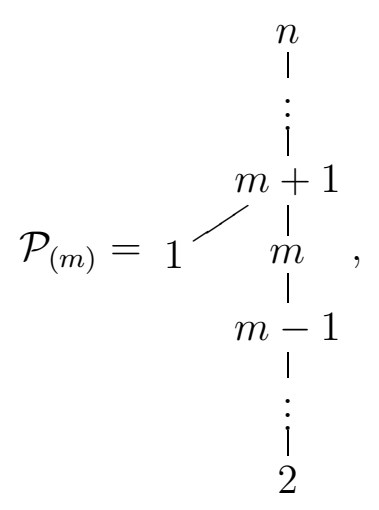

and by convention, let $U_{(1)}=U_{n}$. Note that

$$
U_{n-1} \cong U_{(n)} \triangleleft U_{(n-1)} \triangleleft \cdots \triangleleft U_{(1)}=U_{n} .
$$

The goal of this section is to identify suitable orbit representatives for representatives for $U_{(m)} \backslash \mathfrak{n}_{(m)} / U_{(m)}$ and $U_{(m)} \backslash \mathfrak{n}_{(m)}^{*} / U_{(m)}$.

A matrix $A \in M_{n}\left(\mathbb{F}_{q}\right)$ has an underlying vertex-labeled graph structure $G_{A}$ given by vertices

$$
V_{A}=\left\{A_{i j} \mid 1 \leq i, j \leq n, A_{i j} \neq 0\right\}
$$

and an edge from $A_{i j}$ to $A_{k l}$ if $i=k$ or $j=l$. We label each vertex by its location in the matrix, so $A_{i j}$ has label $(i, j)$. For example, for $a, b, c, d, e, f, g, h \in \mathbb{F}_{q}^{\times}$,

$$
A=\left(\begin{array}{ccccc}
0 & 0 & a & 0 & b \\
c & 0 & 0 & 0 & d \\
0 & e & 0 & f & 0 \\
0 & 0 & 0 & 0 & g \\
0 & 0 & 0 & h & 0
\end{array}\right) \quad \text { implies } \quad G_{A}=\left(\begin{array}{c}
a-b \\
c-f \\
h-f \\
h
\end{array}\right) .
$$




\subsection{Superclass representatives}

Unlike with $U_{n}$, the interpolating groups $U_{(m)}$ have several natural representatives to choose from. In this case, we consider a "natural choice" of an orbit representative to be one with a minimal number of nonzero entries. This section introduces two particular examples.

A matrix $u \in U_{(m)}$ is a comb representative if

(a) At most one connected component of $G_{u-1}$ has more than one element,

(b) If $G_{u-1}$ contains a connected component $S$ with more than one element, then there exist $1 \leq i_{r}<i_{r-1}<\cdots i_{1} \leq m<k_{1}<k_{2}<\cdots<k_{r}$ such that

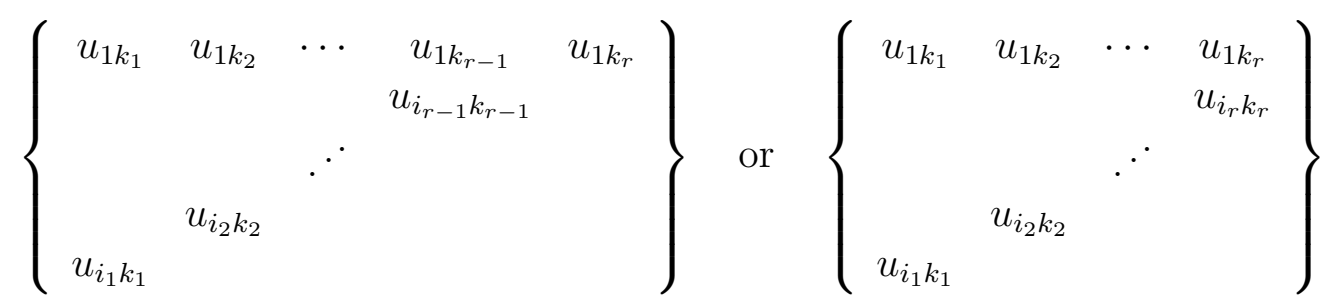

are the vertices of $S$.

A matrix $u \in U_{(m)}$ is a path representative if

(a) At most one connected component of $G_{u-1}$ has more than one element,

(b) If $G_{u-1}$ contains a connected component $S$ with more than one element, then there exist $1<i_{r^{\prime}}<i_{r^{\prime}-1}<\cdots<i_{1} \leq m<k_{1}<k_{2}<\cdots<k_{r}$ with $r^{\prime} \in\{r, r-1\}$ such that

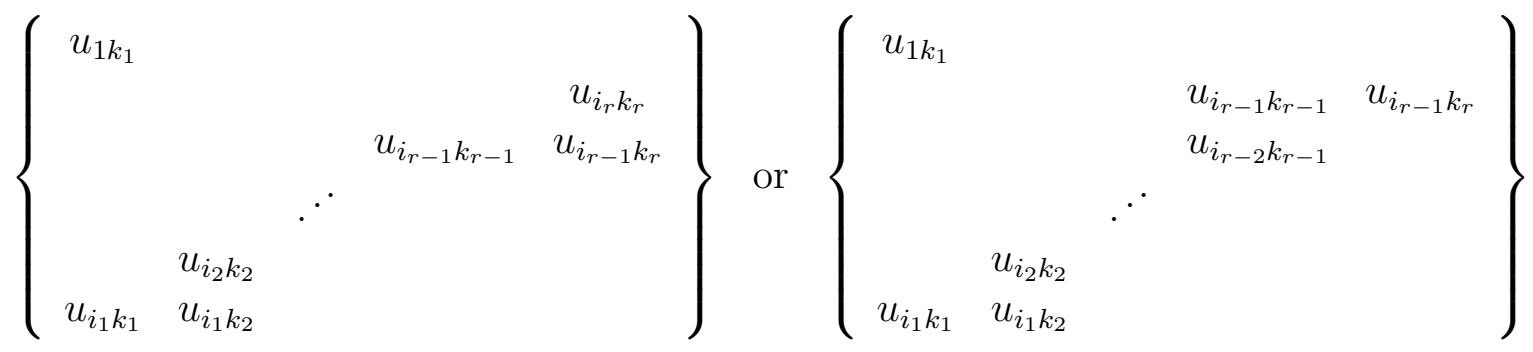

are the vertices of $S$.

Let

$$
\begin{aligned}
& \mathcal{T}_{(m)}^{\vee}=\left\{u \in U_{(m)} \mid u \text { a comb representative }\right\} \\
& \mathcal{Z}_{(m)}^{\vee}=\left\{u \in U_{(m)} \mid u \text { a path representative }\right\} .
\end{aligned}
$$

Let $u \in \mathcal{Z}_{(m)}^{\vee}$. If $G_{u-1}$ has a connected component $S_{u}$ with a vertex in the first row, then we can order the vertices of $S_{u}$ by starting with the vertex in the first row and then 
numbering in order along the path. For example, if

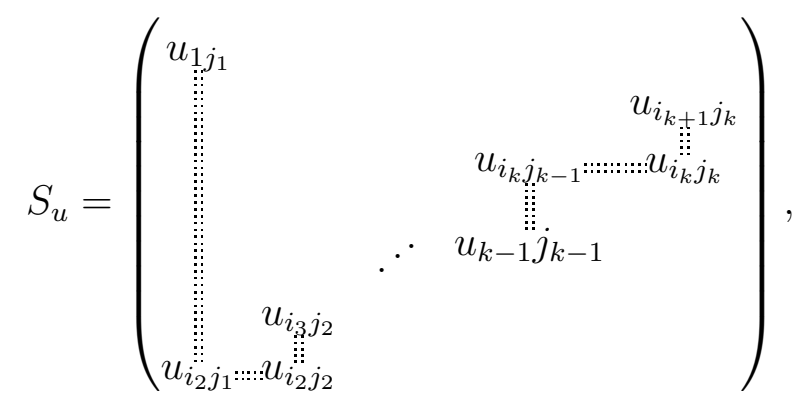

then the order of the vertices is $S_{u}=\left(u_{1 j_{1}}, u_{i_{2} j_{1}}, u_{i_{2} j_{2}}, \ldots, u_{i_{k+1} j_{k}}\right)$. For $i<j$ in $\mathcal{P}$, define the baggage $\operatorname{bag}_{i j}: \mathcal{Z}_{(m)}^{\vee} \rightarrow \mathbb{F}_{q}$ by the rule,

$\operatorname{bag}_{i j}(u)= \begin{cases}u_{i j} x_{k}\left(-x_{k-1}\right)^{-1} x_{k-2}\left(-x_{k-3}\right)^{-1} \cdots\left((-1)^{k+1} x_{1}\right)^{(-1)^{k+1}}, & \text { if } S_{u}=\left(x_{1}, \ldots, x_{l}\right) \\ 1, & u_{i j}=x_{k+1}, k<l \\ \text { otherwise }\end{cases}$

Thus, the function baggage starts at the $(i, j)$ entry and gives a product over all previous non-zero entries in the same path component. Note that the pairs

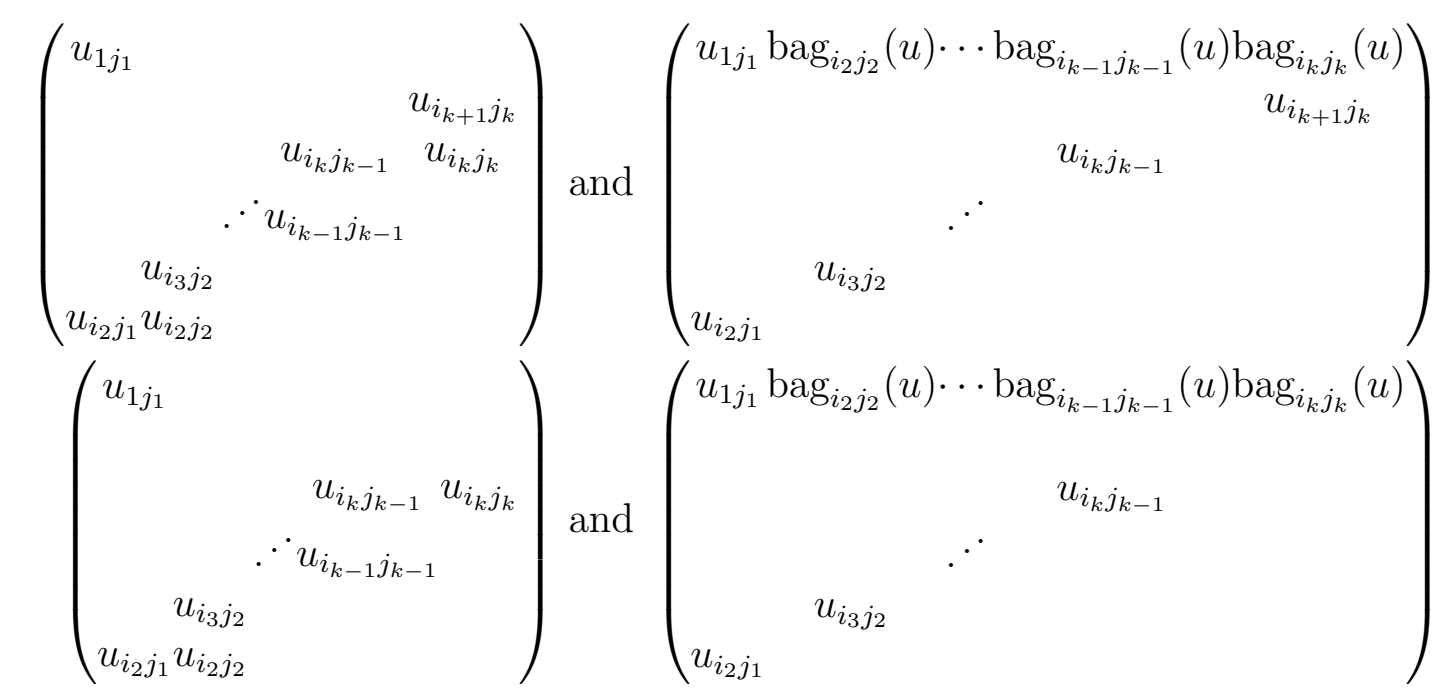

are in the same two sided orbit in $\mathfrak{n}_{\mathcal{P}}$ according to the row and column operations given in Section 2.2.

Proposition 3.1. Let $0<m<n$. Then

(a) $\mathcal{T}_{(m)}^{\vee}$ is a set of superclass representatives for $U_{(m)}$,

(b) $\mathcal{Z}_{(m)}^{\vee}$ is a set of superclass representatives for $U_{(m)}$.

Proof. (a) Let $u \in \mathcal{T}_{(m)}^{\vee}$. Then $U_{(m)}(u-1) U_{(m)} \subseteq U_{n}(u-1) U_{n}$. In fact, if $v \in \mathcal{T}_{(m)}^{\vee}$, but $(v-1) \notin U_{(m)}(u-1) U_{(m)}$, then $(v-1) \notin U_{n}(u-1) U_{n}$. Thus, distinct elements of $\mathcal{T}_{(m)}^{\vee}$ correspond to distinct superclasses of $U_{(m)}$. 
Let $u \in U_{(m)}$ and let $U_{n-1} \subseteq U_{(m)}$ be the subgroup of $U_{n}$ obtained by taking the last $n-1$ rows and columns. Then $U_{n-1}(u-1) U_{n-1} \subseteq U_{(m)}(u-1) U_{(m)}$. We may choose $(v-1) \in U_{n-1}(u-1) U_{n-1}$ such that

(a) every row of $(v-1)$ except row 1 has at most one nonzero entry,

(b) every column of $(v-1)$ has at most two nonzero entries,

(c) if a column has two nonzero entries, then one of the entries must be in the first row.

We may now apply additional row operations allowable by $\mathcal{P}_{(m)}$ to obtain $\left(v^{\prime}-1\right) \in$ $U_{(m)}(u-1) U_{(m)}$, to replace (c) by

(c') if a column has two nonzero entries, then one entry must be in the first row and the second in a row $\leq m$.

Therefore it suffices to show that if the rows of the second nonzero entries do not decrease as we move from left to right, we can convert them into an appropriate form. The following sequence of row and column operations effects such an adjustment.

$$
\begin{gathered}
\left(\begin{array}{cc}
u_{1 k} & u_{1 l} \\
u_{i k} & 0 \\
0 & u_{j l}
\end{array}\right) \stackrel{-u_{1 k}^{-1} u_{1 l} \operatorname{Col}(k)+\operatorname{Col}(l)}{\longrightarrow}\left(\begin{array}{cc}
u_{1 k} & 0 \\
u_{i k} & -u_{i k} u_{1 k}^{-1} u_{1 l} \\
0 & u_{j l}
\end{array}\right) \\
u_{j l}^{-1} u_{i k} u_{1 k}^{-1} u_{l l} \operatorname{Row}(j)+\operatorname{Row}(i) \\
\longrightarrow
\end{gathered}
$$

(b) follows from (a) and (5).

\subsection{Supercharacter representatives}

Recall that we identify $\lambda \in \mathfrak{n}_{\mathcal{P}}^{*}$ with matrices $[\lambda] \in M_{n}\left(\mathbb{F}_{q}\right) / \mathfrak{n}_{\mathcal{P}}^{\perp}$ via the map (1). A function $\lambda \in \mathfrak{n}_{(m)}^{*}$ is a comb representative if

(a) At most one connected component of $G_{[\lambda]}$ has more than one element,

(b) If $G_{[\lambda]}$ has a connected component $S$ with more than one element, then there exist $k_{1}>k_{2}>\cdots>k_{r}>m \geq i_{r^{\prime}}>i_{r^{\prime}-1}>\cdots>i_{1}>1$ with $r^{\prime} \in\{r, r-1\}$ such that

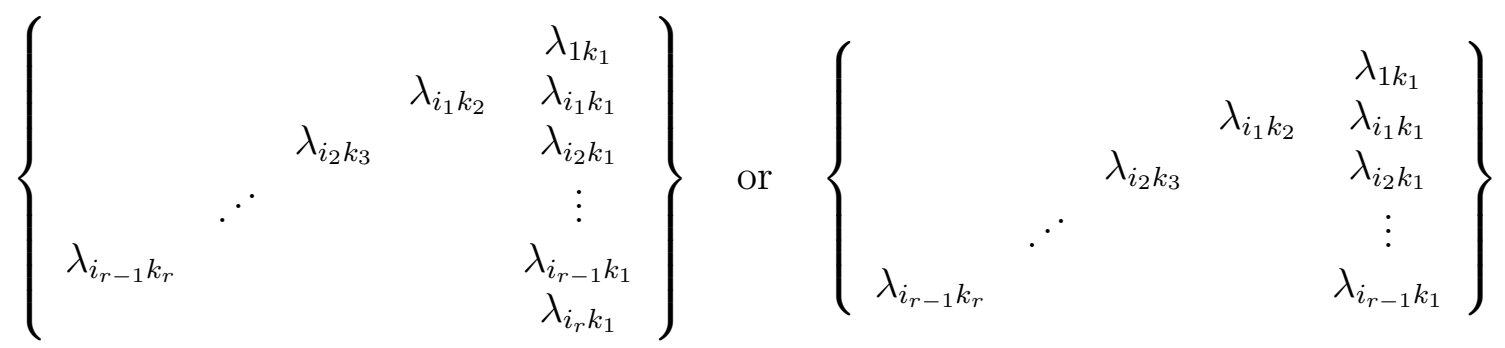

are the vertices of $S$. 
A function $\lambda \in \mathfrak{n}_{(m)}^{*}$ is a path representative if

(a) At most one connected component of $G_{[\lambda]}$ has more than one element,

(b) If $G_{[\lambda]}$ contains a connected component $S$ with more than one element, then there exist $k_{1}>k_{2}>\cdots>k_{r}>m \geq i_{r^{\prime}}>i_{r^{\prime}-1}>\cdots>i_{1}>1$ with $r^{\prime} \in\{r, r-1\}$ such that

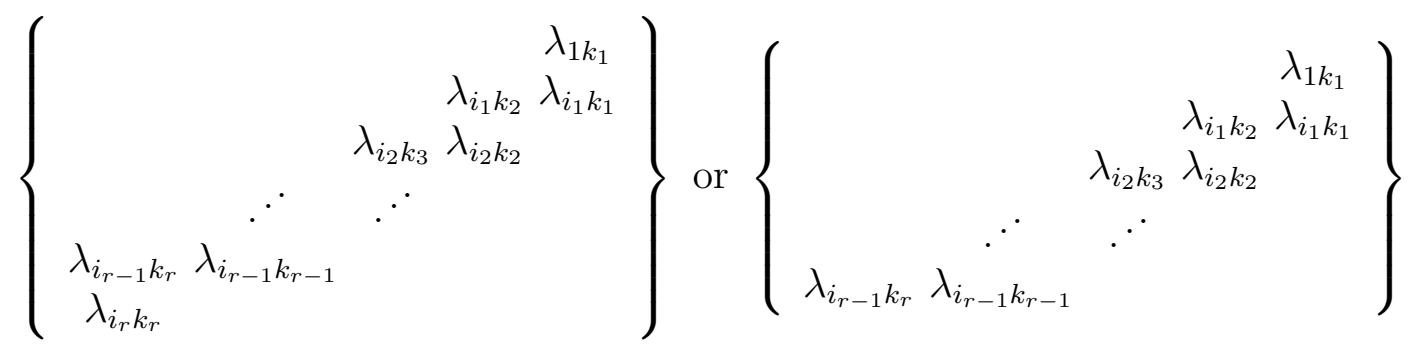

are the vertices of $S$.

Let

$$
\begin{aligned}
& \mathcal{T}_{(m)}=\left\{\lambda \in \mathfrak{n}_{(m)}^{*} \mid \lambda \text { a comb representative }\right\} \\
& \mathcal{Z}_{(m)}=\left\{\lambda \in \mathfrak{n}_{(m)}^{*} \mid \lambda \text { a path representative }\right\} .
\end{aligned}
$$

Let $\lambda \in \mathcal{Z}_{(m)}$. If $G_{[\lambda]}$ has a connected component $S_{\lambda}$ with a vertex in the first row, then we can order the vertices of $S_{\lambda}$ by starting with the vertex in the first row and then numbering in order along the path. For example, if

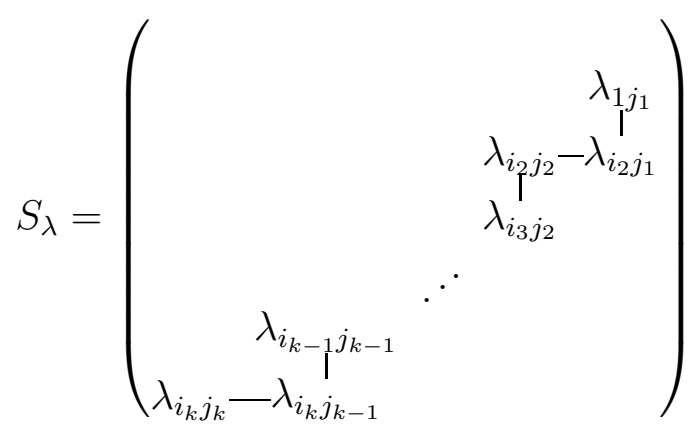

then the order of the vertices is $S_{\lambda}=\left(\lambda_{1 j_{1}}, \lambda_{i_{2} j_{1}}, \lambda_{i_{2} j_{2}}, \ldots, \lambda_{i_{k} j_{k}}\right)$. For $i<j$ in $\mathcal{P}$, define the baggage $\operatorname{bag}_{i j}: \mathcal{Z}_{(m)} \rightarrow \mathbb{F}_{q}$ by the rule,

$$
\operatorname{bag}_{i j}(\lambda)= \begin{cases}\lambda_{i j} y_{k}\left(-y_{k-1}\right)^{-1} y_{k-2}\left(-y_{k-3}\right)^{-1} \cdots\left((-1)^{k+1} y_{1}\right)^{(-1)^{k+1}}, & \text { if } S_{\lambda}=\left(y_{1}, \ldots, y_{l}\right) \\ 1, & \lambda_{i j}=y_{k+1}, k<l \\ & \text { otherwise }\end{cases}
$$


Note that the pairs

$$
\begin{aligned}
& \left(\begin{array}{cc} 
& \lambda_{1 j_{1}} \\
& \lambda_{i_{2} j_{2}} \lambda_{i_{2} j_{1}} \\
& \cdot \cdot \lambda_{i_{3} j_{2}} \\
\lambda_{i_{k-1} j_{k-1}} &
\end{array}\right) \text { ( } \\
& \left(\begin{array}{rr}
\lambda_{1 j_{1}} \\
\\
\lambda_{i_{2} j_{2}} \lambda_{i_{2} j_{1}} \\
\cdot \cdot \lambda_{i_{3} j_{2}} \\
\lambda_{i_{k-1} j_{k-1}} &
\end{array}\right) \text { and } \begin{array}{cc}
\lambda_{1 j_{1}} \\
\\
\\
\lambda_{i_{k} j_{k}} \lambda_{i_{k} j_{k-1}}
\end{array}
\end{aligned}
$$

are in the same two sided orbit in $\mathfrak{n}_{\mathcal{P}}^{*}$ according to the row and column operations given in Section 2.2.

Proposition 3.2. Let $0<m<n$. Then

(a) $\mathcal{T}_{(m)}$ is a set of supercharacter representatives,

(b) $\mathcal{Z}_{(m)}$ is a set of supercharacter representatives.

Proof. (a) Let $\lambda \in \mathcal{T}_{(m)}$. Then $U_{(m)} \lambda U_{(m)} \subseteq U_{n} \lambda U_{n}$. In fact, if $\gamma \in \mathcal{T}_{(m)}$, but $\gamma \notin$ $U_{(m)} \lambda U_{(m)}$, then $\gamma \notin U_{n} \lambda U_{n}$. Thus, distinct elements of $\mathcal{T}_{(m)}$ correspond to distinct twosided orbits in $\mathfrak{n}_{\mathcal{P}}^{*}$.

Since 1 is incomparable to $j \in\{2,3, \ldots, m\}$ in $\mathcal{P}_{(m)}$, we may not add row 1 to row $j$ if $j \leq m$ when computing two-sided orbits. Let $\lambda \in \mathfrak{n}_{\mathcal{P}}^{*}$ and let $U_{n-1} \subseteq U_{(m)}$ be the subgroup of $U_{n}$ obtained by taking the last $n-1$ rows and columns. Then $U_{n-1} \lambda U_{n-1} \subseteq U_{(m)} \lambda U_{(m)}$. We may choose $\gamma \in U_{n-1} \lambda U_{n-1}$ such that

(a) every row of $\gamma$ except row 1 has at most one nonzero entry,

(b) every column of $\gamma$ has at most two nonzero entries,

(c) if a column has two nonzero entries, then one of the entries must be in the first row. We may now apply additional row operations allowable by $\mathcal{P}_{(m)}$ to obtain $\gamma^{\prime} \in U_{(m)} \lambda U_{(m)}$, to replace $(\mathrm{a}),(\mathrm{b}),(\mathrm{c})$ by

(a') every column of $\gamma$ except some column $k$ has at most 1 nonzero entry,

(b') every row has of $\gamma$ has at most two nonzero entries, and row 1 has at most 1 ,

$\left(c^{\prime}\right)$ if a row has two nonzero entries, then one entry must be in column $k$ and the second in a column $j$ such that $m<j<k$.

We can now readjust the nonzero entries to be in an appropriate arrangement as in the proof of Proposition 3.1.

(b) follows from (a) and (6). 


\section{Supercharacter formulas for $U_{(m)}$}

This section develops supercharacter formulas for both comb and path representatives. After developing tools that allow us to decompose characters as products of simpler characters, we prove a character formula for comb characters. We then use the translation between comb and path representatives of (5) and (6) to get a more combinatorial character formula for path representatives.

\subsection{Multiplicativity of supercharacter formulas}

In this section we begin with the general pattern group setting, so let $\mathcal{P}$ be a poset.

Let $u \in U_{\mathcal{P}}$. For a connected component $S$ of $G_{u-1}$, let $[S] \in \mathfrak{n}_{\mathcal{P}}$ be given by

$$
[S]_{j k}= \begin{cases}u_{j k}, & \text { if } u_{j k} \in V_{S} \\ 0, & \text { otherwise }\end{cases}
$$

Similarly, let $\lambda \in \mathfrak{n}_{(m)}^{*}$. For a connected component $T$ of $G_{[\lambda]}$, let $[T] \in M_{n}\left(\mathbb{F}_{q}\right) / \mathfrak{n}_{\mathcal{P}}^{\perp}$ be given by

$$
[T]_{j k}= \begin{cases}\lambda_{j k}, & \text { if } \lambda_{j k} \in V_{T} \\ 0, & \text { otherwise }\end{cases}
$$

The following lemma allows us to decompose the supercharacter formula of a pattern group $U_{\mathcal{P}}$ by connected components.

Lemma 4.1. Let $u \in U_{\mathcal{P}}$ and $\lambda \in \mathfrak{n}_{\mathcal{P}}^{*}$. Let $S_{1}, S_{2}, \ldots, S_{k}$ be the connected components of $G_{u-1}$ and $T_{1}, T_{2}, \ldots, T_{l}$ be the connected components of $G_{[\lambda]}$. Then

$$
\chi^{\lambda}(u)=\prod_{j=1}^{l} \chi^{\left[T_{j}\right]}(1) \prod_{i=1}^{k} \frac{\chi^{\left[T_{j}\right]}\left(\left[S_{i}\right]+1\right)}{\chi^{\left[T_{j}\right]}(1)} .
$$

Proof. Let $U=U_{\mathcal{P}}$. The proof follows from the following two claims:

(1) If $\lambda$ has two components $T$ and $T^{\prime}$, then

$$
\chi^{\lambda}(u)=\chi^{[T]}(u) \chi^{\left[T^{\prime}\right]}(u)
$$

(2) If $u$ has two components $S$ and $S^{\prime}$, then

$$
\chi^{\lambda}(u)=\chi^{\lambda}(1) \frac{\chi^{\lambda}([S])}{\chi^{\lambda}(1)} \frac{\chi^{\lambda}\left(\left[S^{\prime}\right]\right)}{\chi^{\lambda}(1)} .
$$

(1) Note that since $T$ and $T^{\prime}$ involve distinct rows and columns, the left orbits of $[T]$ and $\left[T^{\prime}\right]$ are independent and involve distinct rows. Thus,

$$
|U \lambda|=|U[T]|\left|U\left[T^{\prime}\right]\right|
$$


In fact, for $\lambda^{\prime} \in U \lambda U$,

$$
\left|\left\{(\gamma, \mu) \in(U[T] U) \times\left(U\left[T^{\prime}\right] U\right) \mid \lambda^{\prime}=\gamma+\mu\right\}\right|=\frac{|U[T] U|\left|U\left[T^{\prime}\right] U\right|}{|U \lambda U|} .
$$

Thus, by definition

$$
\begin{aligned}
\chi^{\lambda}(u) & =\frac{|U \lambda|}{|U \lambda U|} \sum_{\lambda^{\prime} \in U \lambda U} \theta\left(-\lambda^{\prime}(u-1)\right) \\
& =\frac{|U \lambda|}{|U[T] U|\left|U\left[T^{\prime}\right] U\right|} \sum_{\substack{\gamma \in U\left[T^{\prime} U \\
\mu \in U\left[T^{\prime}\right] U\right.}} \theta(-\gamma(u-1)-\mu(u-1)) \\
& =\frac{|U[T]|\left|U\left[T^{\prime}\right]\right|}{|U[T] U|\left|U\left[T^{\prime}\right] U\right|} \sum_{\substack{\gamma \in U[T] U \\
\mu \in U\left[T^{\prime}\right] U}} \theta(-\gamma(u-1)) \theta(-\mu(u-1)) \\
& =\frac{|U[T]|}{|U[T] U|} \sum_{\gamma \in U[T] U} \theta(-\gamma(u-1)) \frac{\left|U\left[T^{\prime}\right]\right|}{\left|U\left[T^{\prime}\right] U\right|} \sum_{\mu \in U\left[T^{\prime}\right] U} \theta(-\mu(u-1)) \\
& =\chi^{[T]}(u) \chi^{\left[T^{\prime}\right]}(u) .
\end{aligned}
$$

(2) For any $u^{\prime}-1 \in U(u-1) U$,

$$
\left|\left\{(v-1, w-1) \in(U[S] U) \times\left(U\left[S^{\prime}\right] U\right) \quad \mid u^{\prime}-1=v-1+w-1\right\}\right|=\frac{|U[S] U|\left|U\left[S^{\prime}\right] U\right|}{|U(u-1) U|} .
$$

We have that

$$
\begin{aligned}
\chi^{\lambda}(u) & =\frac{\chi^{\lambda}(1)}{|U(u-1) U|} \sum_{v-1 \in U(u-1) U} \theta(-\lambda(v-1)) \\
& =\frac{\chi^{\lambda}(1)}{|U[S] U|\left|U\left[S^{\prime}\right] U\right|} \sum_{\substack{v-1 \in U[S] U \\
w-1 \in U\left[S^{\prime}\right] U}} \theta(-\lambda(v-1+w-1)) \\
& =\frac{\chi^{\lambda}(1)}{\chi^{\lambda}(1) \chi^{\lambda}(1)} \frac{\chi^{\lambda}(1)}{|U[S] U|} \sum_{v-1 \in U[S] U} \theta(-\lambda(v-1)) \frac{\chi^{\lambda}(1)}{\left|U\left[S^{\prime}\right] U\right|} \sum_{w-1 \in U\left[S^{\prime}\right] U} \theta(-\lambda(w-1)) \\
& =\chi^{\lambda}(1) \frac{\chi^{\lambda}([S])}{\chi^{\lambda}(1)} \frac{\chi^{\lambda}\left(\left[S^{\prime}\right]\right)}{\chi^{\lambda}(1)},
\end{aligned}
$$

as desired.

Corollary 4.1. Let $u \in U_{\mathcal{P}}$ and $\lambda \in \mathfrak{n}_{\mathcal{P}}^{*}$ with connected components $T_{1}, \ldots, T_{l}$. Then

$$
\chi^{\lambda}(u)=\prod_{i=1}^{l} \chi^{\left[T_{i}\right]}(u)
$$


To obtain character formulas for $U_{(m)}$ we will require a slightly more refined multiplicativity result that depends on the poset structure $\mathcal{P}_{(m)}$ and a choice of comb representatives.

For $u \in U_{(m)}$ and $1 \leq k \leq n$, let $u[k] \in U_{(m)}$ be given by

$$
u[k]_{i j}= \begin{cases}u_{i j}, & \text { if } j \in\{i, k\}, \\ 0, & \text { otherwise. }\end{cases}
$$

That is, $u[k]$ is the same as $u$ on the diagonal and in the $k$ th column, but has zeroes elsewhere. For $\lambda \in \mathfrak{n}_{\mathcal{P}}^{*}$, let $\lambda[u, k] \in \mathfrak{n}_{\mathcal{P}}^{*}$ be given by

$$
[\lambda[u, k]]_{i l}= \begin{cases}\lambda_{i l}, & \text { if } k \leq l \text { and } u_{j k} \neq 0 \text { for some } i \leq j<k, \\ 0, & \text { otherwise. }\end{cases}
$$

That is, $[\lambda[u, k]]$ is the same as $[\lambda]$ weakly NorthEast of the nonzero entries of $u$ in the $k$ th column, but has zeroes elsewhere.

The following lemma states that we can compute supercharacter formulas for $U_{(m)}$ column by column on the superclasses.

Lemma 4.2. Let $u \in U_{(m)}$ with $u \in \mathcal{T}_{(m)}^{\vee}$ and let $\lambda \in \mathcal{T}_{(m)}$. Then

(a) The character $\chi^{\lambda}(u) \neq 0$ if and only if $\chi^{\lambda[u, k]}(u[k]) \neq 0$ for all $2 \leq k \leq n$.

(b) The character value

$$
\chi^{\lambda}(u)=\chi^{\lambda}(1) \prod_{k=2}^{n} \frac{\chi^{\lambda[u, k]}(u[k])}{\chi^{\lambda[u, k]}(1)} .
$$

Proof. (a) Let $M$ correspond to $(\lambda, u)$ as in Theorem 2.1. Note that $M_{(i, j),(k, l)}, M_{(i, j),\left(k^{\prime}, l^{\prime}\right)} \in$ $\mathbb{F}_{q}^{\times}$implies $\lambda_{i l}, u_{j k}, \lambda_{i l^{\prime}}, u_{j k^{\prime}} \in \mathbb{F}_{q}^{\times}$, so

$$
u={ }_{j}\left(\begin{array}{ccc} 
& k & k^{\prime} \\
u_{j k} & u_{j k^{\prime}}
\end{array}\right) \quad \text { and } \quad[\lambda]={ }^{i}\left(\begin{array}{rr}
\lambda_{i l} & \lambda_{i l^{\prime}} \\
&
\end{array}\right.
$$

However, since $u \in \mathcal{T}_{(m)}^{\vee}$, the only row of $u$ which can have more than one nonzero entry is row 1 . Since $i<j$, we have $k=k^{\prime}$ and the nonzero entries of $u$ contribute to distinct rows of $M$. Similiarly, if $M_{(i, j),(k, l)}, M_{\left(i^{\prime}, j^{\prime}\right),(k, l)} \in \mathbb{F}_{q}^{\times}$implies $\lambda_{i l}, u_{j k}, \lambda_{i^{\prime} l}, u_{j^{\prime} k} \in \mathbb{F}_{q}^{\times}$, so

$$
u=\underset{j}{j}\left(\begin{array}{c}
k \\
u_{j k} \\
u_{j^{\prime} k}
\end{array}\right) \quad \text { and } \quad[\lambda]={ }_{i^{\prime}}^{i}\left(\begin{array}{c}
l \\
\lambda_{i l} \\
\lambda_{i^{\prime} l}
\end{array}\right) .
$$

Thus, distinct columns of $u$ contribute to distinct columns of $M$. For $1 \leq k \leq n$,

$$
\begin{aligned}
R_{k}= & \begin{array}{l}
\text { rows of } M \text { that have nonzero entries corresponding } \\
\text { to the nonzero entries of } u \text { in column } k
\end{array} \\
C_{k}= & \begin{array}{l}
\text { columns of } M \text { that have nonzero entries corresponding } \\
\text { to the nonzero entries of } u \text { in column } k .
\end{array}
\end{aligned}
$$


By choosing an appropriate order on the rows and columns of $M$,

$$
M=M_{R_{1}, C_{1}} \oplus M_{R_{2}, C_{2}} \oplus \cdots \oplus M_{R_{n}, C_{n}}
$$

where $M_{R_{k}, C_{k}}$ is the submatrix of $M$ using rows $R_{k}$ and columns $C_{k}$.

Using (8), there exists a solution to $M x=-a$ if and only if for each $1 \leq k \leq n$, there exist $x_{k} \in \mathbb{F}_{q}^{\left|C_{k}\right|}$ such that $M_{R_{k}, C_{k}} x_{k}=-a_{R_{k}}$.

If $a_{i j} \neq 0$, then there exist $\lambda_{i k}, u_{j k} \in \mathbb{F}_{q}^{\times}$for some $k$. Since row $j$ in $u$ has at most one nonzero entry, $a_{i j}=u_{j k} \lambda_{i k}$. Thus, $a_{R_{k}}$ only depends on the pair $(\lambda[u, k], u[k])$.

By (8), we have

$$
\operatorname{Null}(M)=\operatorname{Null}\left(M_{R_{1}, C_{1}}\right) \oplus \operatorname{Null}\left(M_{R_{2}, C_{2}}\right) \oplus \cdots \oplus \operatorname{Null}\left(M_{R_{n}, C_{n}}\right),
$$

so $b$ is perpendicular to $\operatorname{Null}(M)$ if and only if $b_{C_{k}}$ is perpendicular to $M_{R_{k}, C_{k}}$ for all $k$. The condition $(k, l) \in C_{k}$ implies $u_{j k} \neq 0$ for some $j$, so $b_{k l} \in \mathbb{F}_{q}^{\times}$implies $b_{k l}=u_{1 k} \lambda_{1 l}+u_{j k} \lambda_{j l}$. Thus, $b_{C_{k}}$ only depends on the pair $(\lambda[u, k], u[k])$, and (a) follows.

(b) Since $C_{1}=R_{1}=\emptyset$, it follows from (8) that

$$
\operatorname{rank}(M)=\sum_{k=1}^{n} \operatorname{rank}\left(M_{R_{k}, C_{k}}\right)=\sum_{k=2}^{n} \operatorname{rank}\left(M_{R_{k}, C_{k}}\right) .
$$

By (a),

$$
\theta(x \cdot b)=\prod_{k=2}^{n} \theta\left(x_{C_{k}} \cdot b_{C_{k}}\right)
$$

and by inspection

$$
\theta \circ \lambda(u-1)=\prod_{(j, k)} \theta\left(u_{j k} \lambda_{j k}\right)=\prod_{k=1}^{n} \prod_{j<k} \theta\left(u_{j k} \lambda_{j k}\right)=\prod_{k=1}^{n} \theta(\lambda[u, k](u[k]-1)) .
$$

Now (b) follows from (a).

Remark. This lemma depends on the choice of representatives. In particular, it is not true for path representatives.

\subsection{A character formula for comb representatives}

It follows from Lemmas 4.1 and 4.2 that to give the character value $\chi^{\lambda}(u)$, we may assume $u-1$ has nonzero entries in one column and $G_{[\lambda]}$ has one connected component $S$.

Theorem 4.1. Let $u \in U_{(m)}$ such that $u \in \mathcal{T}_{(m)}^{\vee}$ and $u-1$ has support $\operatorname{supp}(u-1) \subseteq$ $\{(1, k),(j, k)\}$. Let $\lambda \in \mathcal{T}_{(m)}$ be such that $\lambda$ has one connected component $S$ with $\operatorname{Cols}(S)=$ $\left\{l_{1}<l_{2}<\cdots<l_{s}\right\}$. Then 
(a) Let $i_{1}>i_{2}>\ldots>i_{s-1}$ be such that $\lambda_{i_{d} l_{d}} \neq 0$ for $1 \leq d \leq s-1$. The character degree

$$
\chi^{\lambda}(1)= \begin{cases}q^{l_{s}-m-2} \prod_{d=1}^{s-1} q^{l_{d}-i_{d}-1}, & \text { if } \lambda_{i l_{s}}=0 \text { for all } i>i_{1}, \\ q^{l_{s}-i-1} \prod_{d=1}^{s-1} q^{l_{d}-i_{d}-1}, & \text { if } \lambda_{i l_{s}} \neq 0 \text { for some } i>i_{1} .\end{cases}
$$

(b) The character

$$
\chi^{\lambda}(u)=0
$$

unless at least one of the following occurs

(1) $u_{j k} \lambda_{i k} \neq 0$ implies $i=1$ with $j \leq m$ or $i>j$; and $u_{1 k} \lambda_{1 l}+u_{j k} \lambda_{j l}=0$ for all $j<k<l$,

(2) $u_{j k} \lambda_{i k} \neq 0$ for some $1<i<j \leq m$, but $\left|R_{k}\right|=\left|C_{k}\right|>0\left(R_{k}\right.$ and $C_{k}$ are as in (7)),

(3) $u_{1 k} \lambda_{1 l_{s}}+u_{j k} \lambda_{j l} \neq 0$ for some $m<k<l$, but $\lambda_{i k}=0$ for all $i$ and $\left|R_{k}\right| \geq\left|C_{k}\right|>0$,

(4) $u_{j k}, \lambda_{j l_{s}}, \lambda_{i l_{s}} \in \mathbb{F}_{q}^{\times}$with $i<j<k<l_{s}$ with $\lambda_{j k^{\prime}}=0$ for all $k<k^{\prime}<l_{s}$.

(c) The character values are

$$
\chi^{\lambda}(u)= \begin{cases}\frac{\chi^{\lambda}(1)}{q^{\left|C_{k}\right|-\delta_{R C}}} \theta\left(u_{j k} \lambda_{j k}\right), & \text { if (1) } \\ \frac{\chi^{\lambda}(1)}{q^{\left|C_{k}\right|}}, & \text { if (2) or (3) or (4) } \\ \frac{\chi^{\lambda}(1)}{q^{\left|C_{k}\right|}} \theta\left(-\lambda_{i l_{s}}^{-1} \lambda_{i k}\left(u_{1 k} \lambda_{1 l_{s}}+u_{j k} \lambda_{j l_{s}}\right)\right), & \text { if (2) and (4), }\end{cases}
$$

where $\delta_{R C}=1$ if $\left|C_{k}\right|>\left|R_{k}\right|$ and $\delta_{R C}=0$ if $\left|C_{k}\right| \leq\left|R_{k}\right|$.

Proof. (a) This is just a statement of the fact that

$$
\chi^{\lambda}(1)=\left|U_{(m)} \lambda\right|
$$

combined with the structure of $S$.

(b) and (c). Note that by Lemma 4.2,

$$
\chi^{\lambda}(u)=\frac{\chi^{\lambda}(1)}{\chi^{\lambda[u, k]}(1)} \chi^{\lambda[u, k]}(u[k]),
$$

so we may assume $M=M_{R_{k}, C_{k}}($ see $(8))$. Let $\operatorname{Rows}(S)=\left\{i_{1}>\ldots>i_{s}\right\}$ or $\operatorname{Rows}(S)=$ $\left\{i_{0}>i_{1}>\ldots>i_{s}\right\}$ be the rows with nonzero entries in $S$ such that $\lambda_{i_{d} l_{d}}, \lambda_{i_{d} l_{s}} \in \mathbb{F}_{q}^{\times}$, and, 
if $i_{0} \in \operatorname{Rows}(S)$, then $\lambda_{i_{0} l_{s}} \neq 0$ (see the definition of comb representatives in Section 3.2). Let $r$ and $r^{\prime}$ be minimal such that $l_{r}>k$ and $i_{r^{\prime}}<j$. Then

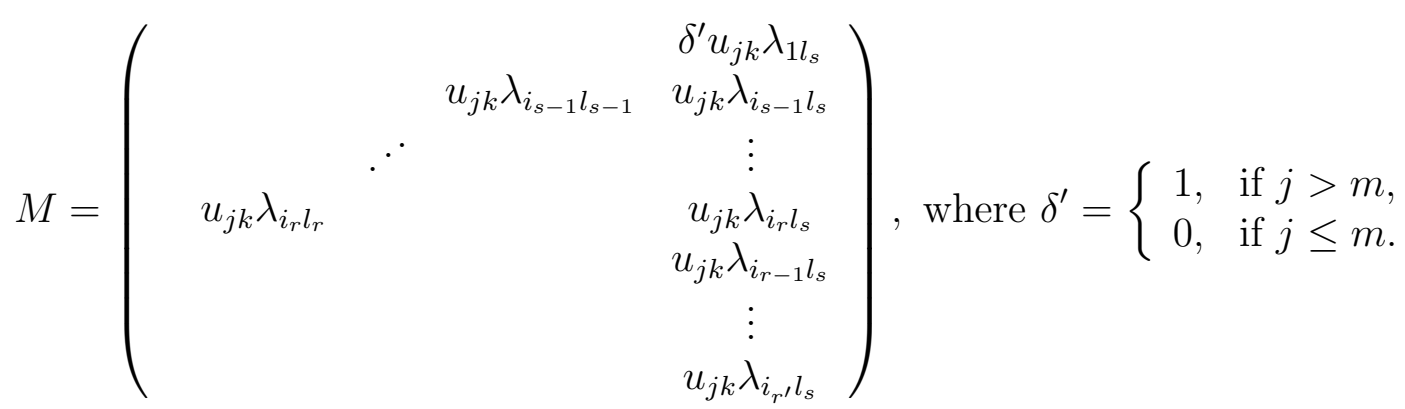

Thus, the rank of $M$ is $q^{\left|C_{k}\right|-\delta_{R C}}$.

Furthermore, $a \in \mathbb{F}_{q}^{\left|R_{k}\right|}$ and $b \in \mathbb{F}_{q}^{\left|C_{k}\right|}$ are given by

$$
\begin{array}{ll}
a_{i j}=u_{j k} \lambda_{i k}, & \text { for }(i, j) \in R_{k}, \\
b_{k l}=\left\{\begin{array}{ll}
u_{1 k} \lambda_{1 l}+u_{j k} \lambda_{j l}, & \text { if } l \in \operatorname{Cols}(S), \\
0 & \text { otherwise }
\end{array} \text { for }(k, l) \in C_{k} .\right.
\end{array}
$$

If $a=0$ then $M \cdot 0=-0$ is easily satisfied, and if $b=0$ then $b \cdot \operatorname{Null}(M)=0$ is also trivially satisfied. Thus, $\chi^{\lambda}(u) \neq 0$ if $u_{j k} \lambda_{i k}=0$ for all $i<j<k$ in $\mathcal{P}_{(m)}$ and $u_{1 k} \lambda_{1 l}+u_{j k} \lambda_{j l}=0$ for all $1<j<k<l$. Note that in the poset $\mathcal{P}_{(m)}, 1 \not \leq j$ for $j \leq m$.

Suppose $a_{i j} \neq 0$. Note that $M x=-a$ can only be satisfied if row $(i, j)$ of $M$ has a nonzero element. That is, there exists $i<j<k<l$ such that $\lambda_{i l} \neq 0$. Consequently, we may assume $k<l_{s}$. If $j>m$, then $\delta=1$, so $(M x)_{1 j} \neq 0$ if and only if $(M x)_{i j} \neq 0$. However, $a_{1 j} \neq 0$ and $(M x)_{1 j} \neq 0$ implies the first row of $\lambda$ has two nonzero elements, contradicting the structure of $S$. Thus, if $a_{i j} \neq 0$ and $M x=-a$ for some $x$, then $j \leq m$ and $k<l_{s}$.

Suppose $a_{i j}=u_{j k} \lambda_{i k} \neq 0$ with $j \leq m$ and $k<l_{s}$. By the definition of $M$ and (9), $(i, k)=\left(i_{r-1}, l_{r-1}\right)$. Note that $(M x)_{i j} \neq 0$ if and only if $(M x)_{i_{r^{\prime}} j} \neq 0$. Since $u_{j k^{\prime}}=0$ for all $k^{\prime} \neq k$, in this case $r^{\prime}=r-1$ or $\left|C_{k}\right|=\left|R_{k}\right|$. Then $M x=-a$, where

$$
x_{k l}=\left\{\begin{array}{ll}
-\lambda_{i l_{s}}^{-1} \lambda_{i k}, & \text { if } l=l_{s}, \\
\lambda_{i_{d} l_{d}}^{-1} \lambda_{i_{d}} l_{s} \lambda_{i l_{s}}^{-1} \lambda_{i k}, & \text { if } l=l_{d}, \\
0, & \text { otherwise, }
\end{array} \quad \text { where }(k, l) \in C_{k} .\right.
$$

If $b_{k l} \neq 0$ and $M$ has no nonzero entry in column $(k, l)$, then $b \cdot \operatorname{Null}(M) \neq 0$. Thus, if $b_{k l} \neq 0$ we must have $\lambda_{j l}, \lambda_{i l} \in \mathbb{F}_{q}^{\times}$with $i<j$. In particular, $j \leq m$, and either $u_{1 k}=0$ or $u$ has two nonzero elements. Since only the last column of $S$ can have more than one nonzero entry, $l=l_{s}$, and $b_{k l_{s}}=u_{1 k} \lambda_{1 l_{s}}+u_{j k} \lambda_{j l_{s}}$. Note that

$$
\operatorname{dim}(\operatorname{Null}(M))= \begin{cases}s-r, & \text { if } \delta^{\prime}=0, r^{\prime}=r \\ 0, & \text { otherwise }\end{cases}
$$

It follows that when $b \neq 0$, then $b$ is perpendicular to $\operatorname{Null}(M)$ if and only if $r^{\prime}>r$ if and only if $\left|R_{k}\right| \geq\left|C_{k}\right|$ (if $\delta^{\prime}=1$, then $j>m$ ). 
In the case that $j=i_{r-2}$ and $k=l_{r-1}$, we have

$$
\theta(x \cdot b)=\theta\left(-\lambda_{i l_{s}}^{-1} \lambda_{i k}\left(u_{1 k} \lambda_{1 l_{s}}+u_{j k} \lambda_{j l_{s}}\right)\right), \quad \text { where } i=i_{r-1} .
$$

Otherwise, $\theta(x \cdot b)=1$.

At this point, it may be helpful to give a more visual interpretation of the conditions in Theorem 4.1 by considering the configurations of superimposed graphs $G_{[\lambda]}$ and $G_{u}$. Recall, for $\lambda \in \mathcal{Z}_{(m)} \cup \mathcal{T}_{(m)}$ there is at most one connected component of $G_{[\lambda]}$ that can have more than one element (or can have a vertex in the first row of $\lambda$ ). Therefore, for $\lambda \in \mathcal{Z}_{(m)} \cup \mathcal{T}_{(m)}$, let

$$
\begin{aligned}
S_{\lambda} & =\text { the connected component of } G_{[\lambda]} \text { that has a vertex in the first row } \\
\operatorname{lc}(\lambda) & = \begin{cases}\min \left\{k \mid S_{\lambda} \text { has a vertex in column } k\right\}, & \text { if } S_{\lambda} \neq \emptyset, \\
0, & \text { otherwise, }\end{cases} \\
\operatorname{br}(\lambda) & = \begin{cases}\max \left\{j \mid S_{\lambda} \text { has a vertex in row } j\right\}, & \text { if } S_{\lambda} \neq \emptyset, \\
n, & \text { otherwise, }\end{cases} \\
\operatorname{wt}(\lambda) & = \begin{cases}\#(\text { Nonzero entries in row } \operatorname{br}(\lambda) \text { of } \lambda)-1, & \text { if } S_{\lambda} \neq \emptyset, \\
0, & \text { otherwise. }\end{cases}
\end{aligned}
$$

For example, if

$$
\lambda=\left(\begin{array}{llllll}
0 & 0 & 0 & 0 & 0 & a \\
0 & 0 & 0 & 0 & c & b \\
0 & 0 & 0 & e & 0 & 0 \\
0 & 0 & 0 & 0 & 0 & d \\
0 & 0 & 0 & 0 & 0 & 0 \\
0 & 0 & 0 & 0 & 0 & 0
\end{array}\right), \quad \text { then } \quad S_{\lambda}=c-\begin{array}{ll}
a & \operatorname{lc}(\lambda)=5 \\
\operatorname{br}(\lambda)=4 & \\
\operatorname{wt}(\lambda)=0
\end{array}
$$

In the following discusion, we will suppress the values of the vertices and distinguish between $G_{u-1}$ and $G_{[\lambda]}$ by the following conventions,

\begin{tabular}{|l|c|c|}
\hline & $G_{u-1}$ & $G_{[\lambda]}$ \\
\hline Vertices & $\bullet$ & $\bullet$ \\
\hline Edges & $\bullet \cdots \cdots \bullet$ & $\bullet$ \\
\hline
\end{tabular}

If $\left|S_{\lambda}\right|>1$ and $\operatorname{wt}(\lambda)=0$, then add an edge to the non-zero vertex of row $\operatorname{br}(\lambda)$ that extends West of this vertex,

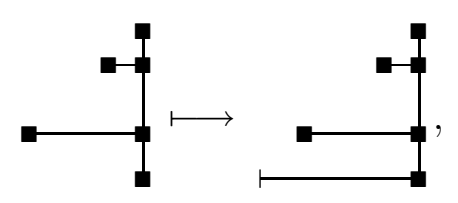

thereby "completing" the comb. 
Vertices of $G_{u-1}$ see North in their column and East in their row, while vertices of $G_{[\lambda]}$ see South in their column and West in their row (in both cases they do not see the location they are in). That is,

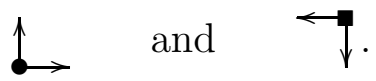

Connected component $S$ of $G_{u-1}$ and $T$ of $G_{[\lambda]}$ see one-another if when one superimposes their matrices, a vertex of $S$ sees a vertex of $T$ (and vice-versa).

The tines of $S_{\lambda}$ are the pairs of horizontal edges with their leftmost vertices. For example, the tines of

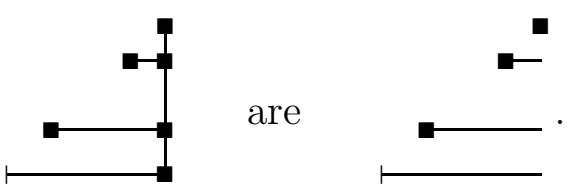

Suppose $u \in U_{(m)}$ has at most two nonzero superdiagonal entries $u_{1 k}, u_{j k} \in \mathbb{F}_{q}$, for some $1 \leq k \leq n$, and suppose $\lambda \in \mathfrak{n}_{(m)}^{*}$ such that $G_{[\lambda]}$ has exactly one connected component $S$. Then column $k$ of $u$ is comb compatible with $S$ if the following conditions are satisfied.

(CC1) If column $k$ of $u$ has exactly one nonzero entry $u_{j k}$ in column $k$ and $S \neq S_{\lambda}$, then $u_{j k}$ cannot see the vertex of $S$,

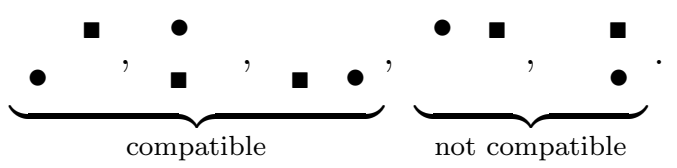

(CC2) If $u_{1 k}, u_{j k} \in \mathbb{F}_{q}^{\times}$, with $1<j$ and $S \neq S_{\lambda}$, then the vertex of $S$ cannot see $u_{1 k}$ or $u_{j k}$,

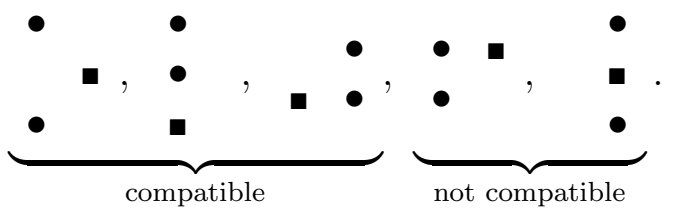

(CC3) If column $k$ of $u$ has exactly one nonzero entry $u_{j k}$ in column $k$ and $S=S_{\lambda}$, then $u_{j k}$ sees a vertex of $S$ if and only if $j \leq m, u_{j k}$ is South of the end of a tine and weakly North of the next tine to the South (if there is another tine),

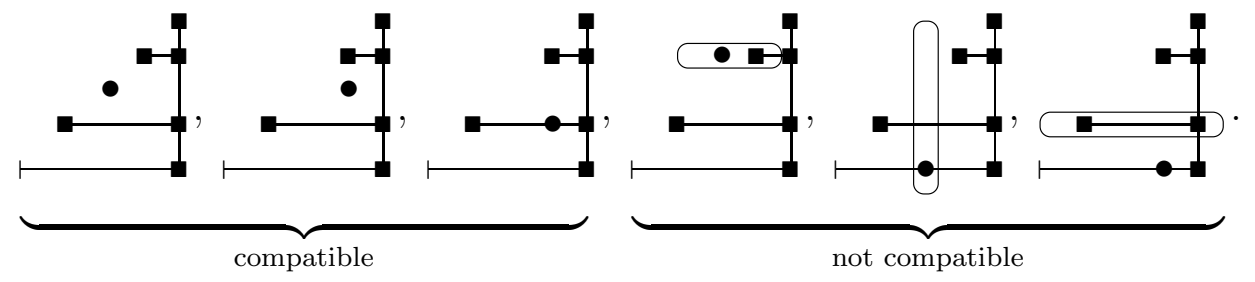


(CC4) If $u_{1 k}, u_{j k} \in \mathbb{F}_{q}^{\times}$, with $1<j$ and $S=S_{\lambda}$, then a vertex of $S$ sees $u_{1 k}$ or $u_{j k}$ if and only if either $u_{j k}$ is not South of the end of a tine but on a tine of $S$, or $u_{j k}$ is South of the end of a tine and weakly North of the next tine to the South,

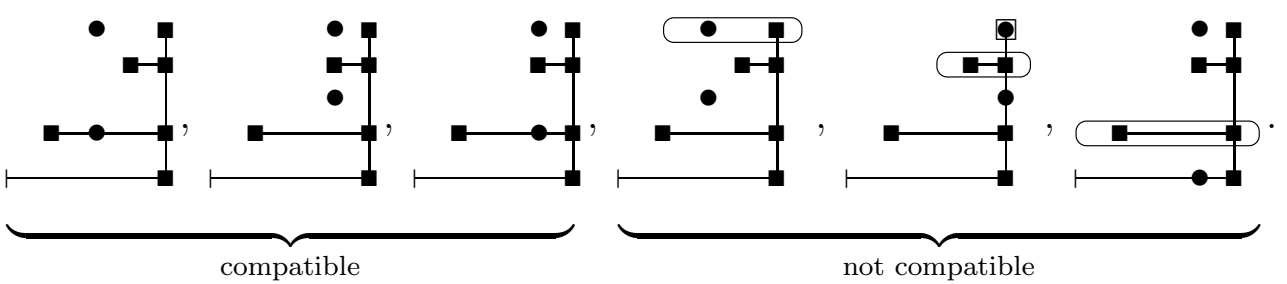

From this point of view, Theorem 4.1 translates to the following corollary.

Corollary 4.2. Let $1 \leq m \leq n$. Suppose $u \in U_{(m)}$ has at most two nonzero superdiagonal entries $u_{1 k}, u_{j k} \in \mathbb{F}_{q}$, for some $1 \leq k \leq n$. For $\lambda \in \mathcal{T}_{(m)}$, suppose $G_{[\lambda]}$ has one connected component $S$. Then

(a) The character degree

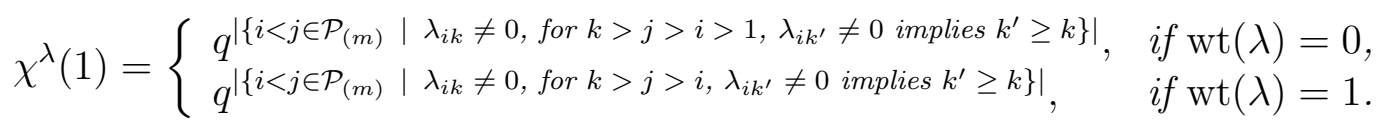

(b) The character

$$
\chi^{\lambda}(u)=0
$$

unless column $k$ of $u$ and $S$ are comb compatible and in condition (CC4) if $u_{1 k}$ sees a vertex $S$ and $u_{j k}$ is not strictly South and weakly East of the end of a tine,

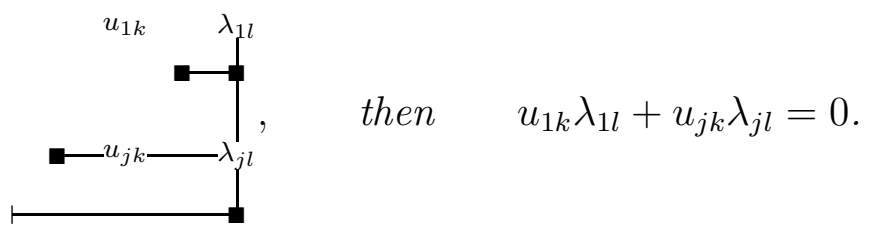

(c) If $\chi^{\lambda}(u) \neq 0$, then

$$
\chi^{\lambda}(u)=\chi^{\lambda}(1) \frac{\theta\left(u_{1 k} \lambda_{1 k}+u_{j k} \lambda_{j k}\right)}{q^{c(u, \lambda)}} \prod_{\substack{i<j<l \\ \lambda_{i l}, \lambda_{j l} \in \mathbb{E}_{q}^{\times}}} \theta\left(-\lambda_{i l}^{-1} \lambda_{i k}\left(u_{1 k} \lambda_{1 l}+u_{j k} \lambda_{j l}\right)\right)
$$

where

$$
c(u, \lambda)= \begin{cases}\mid\left\{l>k \mid \lambda_{i l} \neq 0, \text { for some } i<j\right\} \mid, & \text { if } u_{j k} \neq 0, j>m, \\ \mid\left\{l>k \mid \lambda_{i l} \neq 0, \text { for some } i<j\right\} \mid, & \text { if } u_{j k}, \lambda_{i j^{\prime}} \lambda_{i l} \in \mathbb{F}_{q}^{\times} \text {with } \\ \mid\left\{l>k \mid \lambda_{i l} \neq 0, \text { for some } i<j\right\} \mid-1, & \text { otherwise. }\end{cases}
$$




\subsection{A character formula for path representatives}

For $\lambda \in \mathcal{Z}_{(m)} \cup \mathcal{T}_{(m)}$, let $S_{\lambda}, \operatorname{lc}(\lambda), \operatorname{br}(\lambda)$, and $\operatorname{wt}(\lambda)$ be as in the previous section. For $\lambda \in \mathcal{Z}_{(m)}$, order the vertices of $S_{\lambda}$ starting with the vertex in the first row and proceeding along the path to the vertex in position $(\operatorname{br}(\lambda), \operatorname{lc}(\lambda))$. For example, if

$$
\lambda=\left(\begin{array}{llllll}
0 & 0 & 0 & 0 & 0 & a \\
0 & 0 & 0 & 0 & c & b \\
0 & 0 & 0 & e & 0 & 0 \\
0 & 0 & 0 & 0 & d & 0 \\
0 & 0 & 0 & 0 & 0 & 0 \\
0 & 0 & 0 & 0 & 0 & 0
\end{array}\right), \quad \text { then } \quad S_{\lambda}=\left.\right|_{d} ^{a b}, \begin{array}{ll}
\operatorname{lc}(\lambda)=5 & \operatorname{br}(\lambda)=4 \\
\operatorname{wt}(\lambda)=0 .
\end{array}
$$

Before translating from comb representatives to path representatives, we will add some decorations to the graphs $G_{u-1}$ and $G_{[\lambda]}$ of Section 3. We will again suppress the values of the vertices and distinguish between $G_{u-1}$ and $G_{[\lambda]}$ by the following conventions,

\begin{tabular}{|l|c|c|}
\hline & $G_{u-1}$ & $G_{[\lambda]}$ \\
\hline Vertices & $\bullet$ & $\bullet$ \\
\hline Edges & $\bullet \cdots: \bullet$ & $\bullet$ \\
\hline
\end{tabular}

If $S_{\lambda} \neq \emptyset$, then add an additional edge from the vertex in position $(\operatorname{br}(\lambda), \operatorname{lc}(\lambda))$. If $\operatorname{wt}(\lambda)=0$ and $\left|S_{\lambda}\right| \neq 1$ extend the edge West until it reaches just past the $(m+1)$ th column. If $\operatorname{wt}(\lambda)=1$ or $\left|S_{\lambda}\right|=1$, then extend the edge South until just past the $m$ th row. For example,

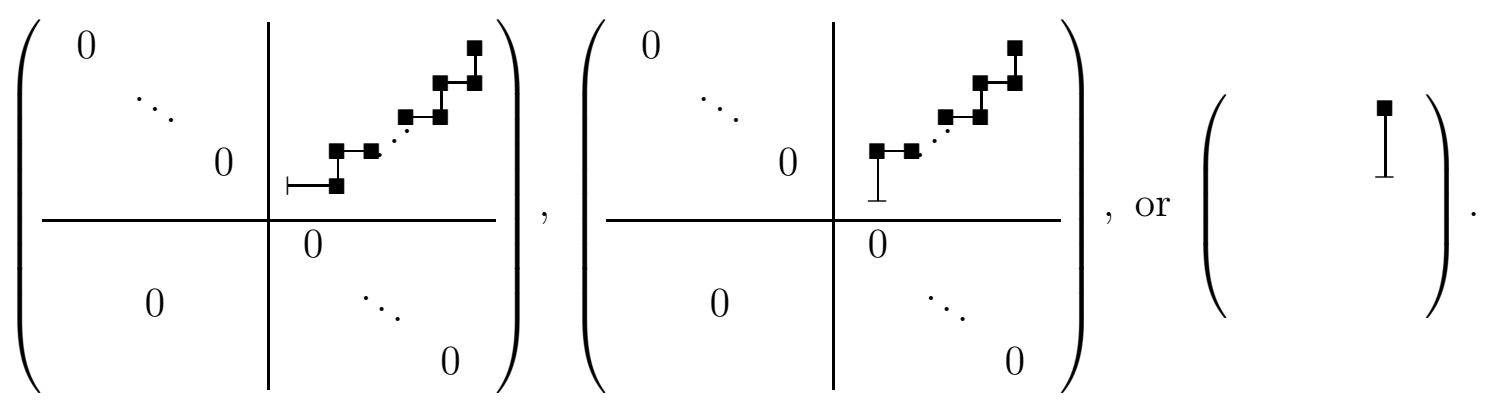

A bottom corner of $\lambda$ is a vertex $v$ in $S_{\lambda}$ with a horizontal edge extending West of $v$. A top corner of $\lambda$ is a vertex $v$ in $S_{\lambda}$ with a vertical edge extending South of $v$. All vertices of $G_{[\lambda]}$ which are not in $S_{\lambda}$ are considered to be both top and bottom corners.

Similarly, if $u \in \mathcal{Z}_{(m)}^{\vee}$, then $G_{u-1}$ has at most one connected component $S_{u}$ that has a vertex in the first row. Order the vertices of $S_{u}$ starting with the vertex in the first row, and proceeding along the path. If $\left|S_{u}\right|>1$, add an edge to $S_{u}$ by extending an edge from the last vertex in the opposite direction of the previous edge (either East or North). Furthermore, if $\left|S_{u}\right|>1$, then view the first edge as not being in the same plane as the matrix, so it no longer crosses any edges that are in the plane of the matrix. Thus, $S_{u}$ 
will be of the form,

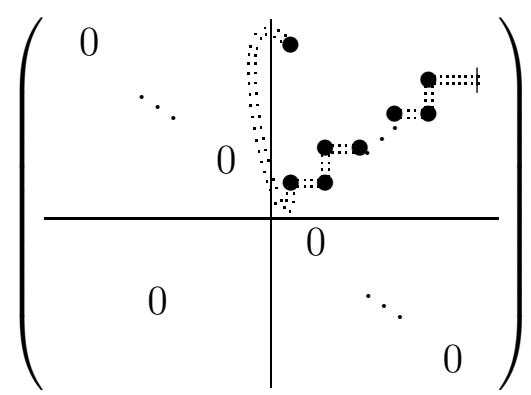

Or

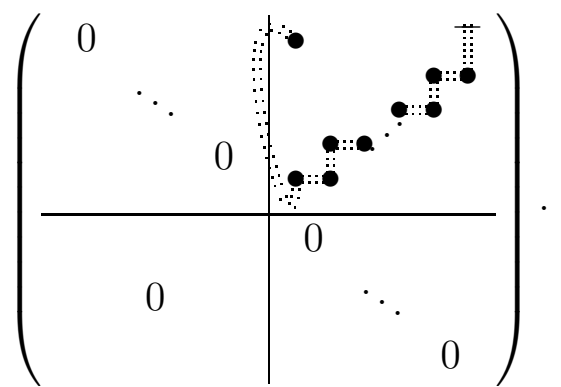

The left corners of $u$ are the leftmost nonzero entries in the rows of $u-1$. The right corners of $u$ are the rightmost nonzero entries in the rows of $u-1$.

Left and right corners see North in their column and East in their row, while top and bottom corners see South in their column and West in their row (in both cases they do not see the location they are in). That is,

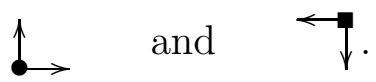

Connected components $S$ of $G_{u}$ and $T$ of $G_{[\lambda]}$ see one-another if when one superimposes their matrices, a corner of $S$ sees a corner of $T$.

Fix $u \in \mathcal{Z}_{(m)}^{\vee}$ and $\lambda \in \mathcal{Z}_{(m)}$ with a connected component $S$ of $G_{u}$ and $T$ of $G_{[\lambda]}$. The components $S$ and $T$ are path compatible if the following conditions are satisfied.

(PC1) If $S \neq S_{u}$ and $T \neq S_{\lambda}$, then $S$ cannot see $T$,

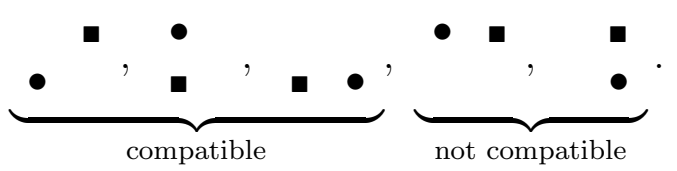

(PC2) If $S=S_{u}$ and $T \neq S_{\lambda}$, then $S$ sees $T$ if and only if $T$ touches a vertical edge of $S$ and no left corner of $S$ sees $T$.
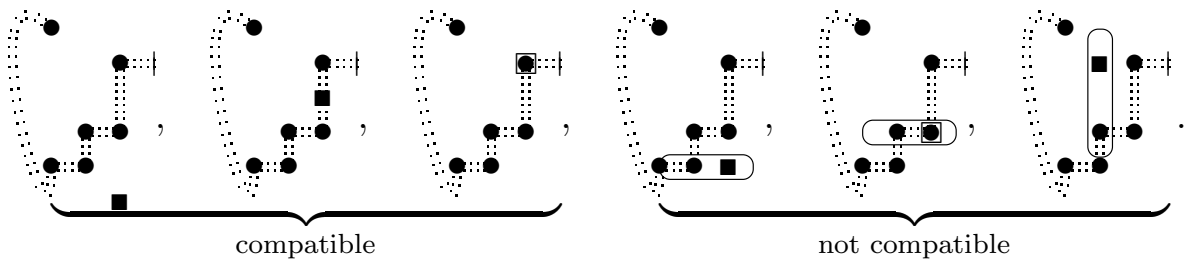

(PC3) If $S \neq S_{u}$ and $T=S_{\lambda}$, then $S$ sees $T$ if and only if $S$ touches a vertical edge of $T$ and no bottom corner of $T$ sees $S$.

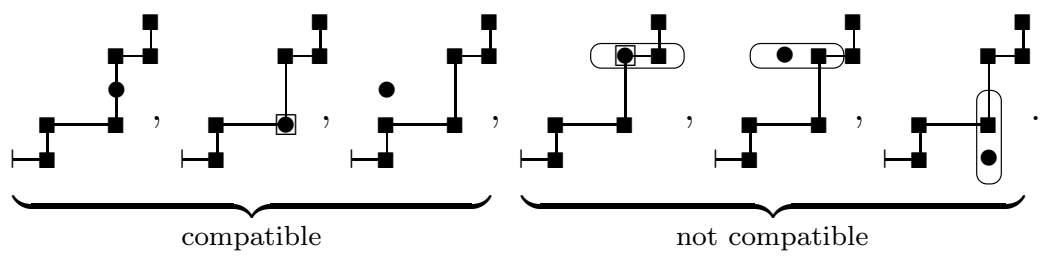


(PC4) If $S=S_{u}$ and $T=S_{\lambda}$, then $S$ sees $T$ if and only if $T$ is never strictly South of $S ; S$ ends weakly East of the beginning of $T$; and left corners of $S$ and bottom corners of $T$ only see one-another horizontally.
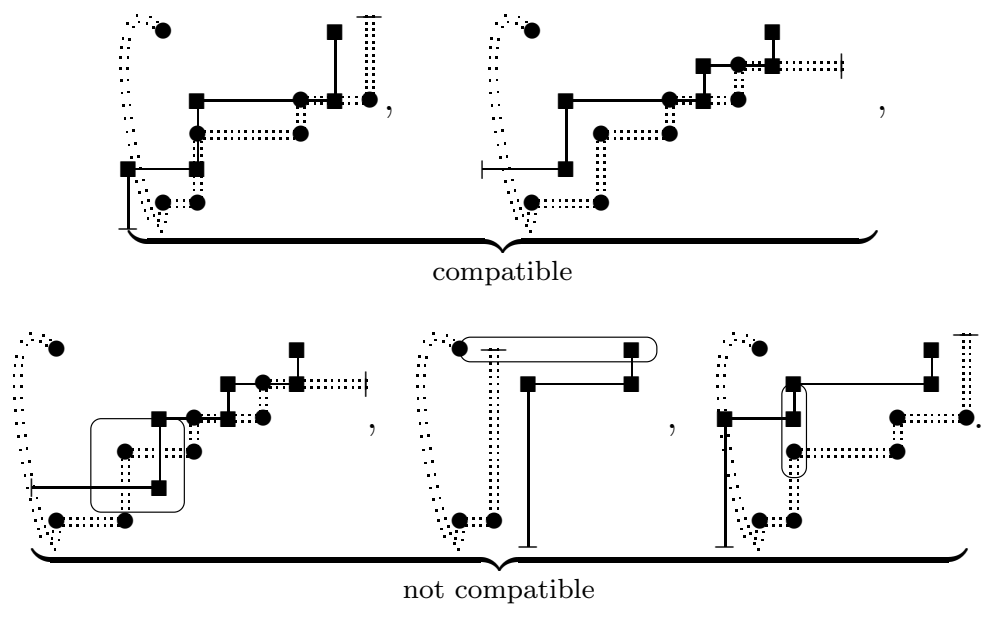

Note that $(\mathrm{PC} 1)-(\mathrm{PC} 4)$ are translations of $(\mathrm{CC} 1)-(\mathrm{CC} 4)$ via the correspondence $(6)$.

Define

$$
\begin{aligned}
& \Theta: \mathcal{Z}_{(m)}^{\vee} \times \mathcal{Z}_{(m)} \longrightarrow \quad \mathbb{C} \\
& (u, \lambda) \quad \mapsto \theta_{E B(u, \lambda)} \prod \theta\left(\operatorname{bag}_{k l}(u) \operatorname{bag}_{i l}(\lambda)\right), \\
& \begin{array}{l}
u_{j l}, u_{k l} \in \mathbb{F}_{q}^{\times} \\
\lambda_{i l}, \lambda_{j l} \in \mathbb{F}_{q}^{\times}
\end{array}
\end{aligned}
$$

where

$$
\theta_{E B(u, \lambda)}= \begin{cases}\theta\left(\operatorname{bag}_{i l}(u) \lambda_{1 l}\right), & \begin{array}{l}
\text { if } \lambda_{1 l} \neq 0, S_{u-1} \text { ends with last } \\
\text { vertex } u_{i l} \text { and a vertical edge }
\end{array} \\
1, & \text { otherwise. }\end{cases}
$$

For a left corner $u_{j k}$ in $u$, let

$$
n_{j k}(\lambda)=\text { Number of bottom corners } \lambda_{i l} \text { with } i<j<k<l \text { in } \mathcal{P}_{(m)} .
$$

Corollary 4.3. Let $u \in U_{(m)}$ be such that $u-1 \in \mathcal{Z}_{(m)}^{\vee}$, and let $\lambda \in \mathcal{Z}_{(m)}$. Then

(a) The character

$$
\chi^{\lambda}(u)=0
$$

unless the connected components of $G_{u-1}$ and $G_{[\lambda]}$ are pairwise path compatible, and in the superimposed matrices, every

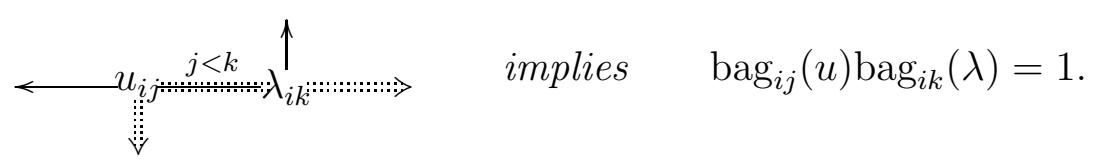


(b) The character degree

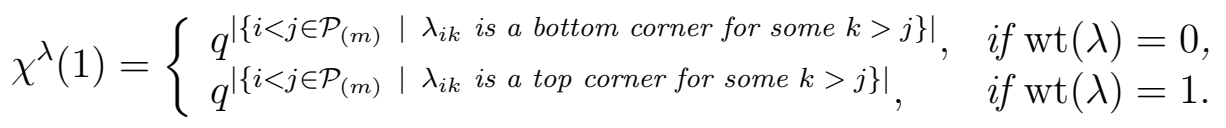

(c) If $\chi^{\lambda}(u) \neq 0$, then

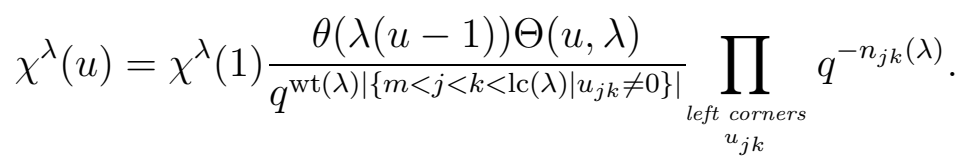

Proof. This corollary follows directly from Corollary 4.2 with the following observations, using (6).

(a) If a bottom corner of $T$ sees a left corner of $S$ horizontally, then we are in the situation of (11), so
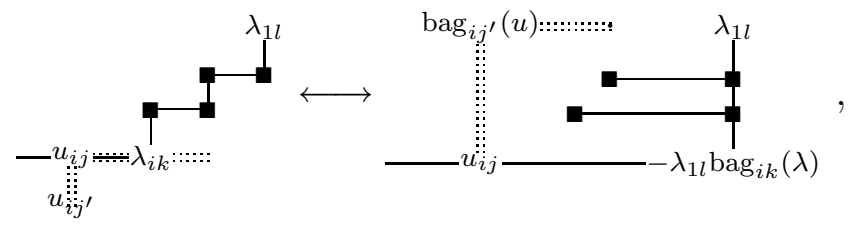

so the comb representations of $\lambda$ and $u$ must satisfy condition (10). However, this is equivalent to $\operatorname{bag}_{i j}(u) \operatorname{bag}_{i k}(\lambda)=1$. Thus, Corollary 4.2 (a) is satisfied if and only if Corollary 4.3 (a) is satisfied.

(b) is straight-forward translation of the combinatorics.

(c) Let $\tilde{\lambda} \in \mathcal{T}_{(m)}$ be the comb representative corresponding to $\lambda \in \mathcal{Z}_{(m)}$ and let $\tilde{u} \in \mathcal{T}_{(m)}^{\vee}$ be the comb representative in the same superclass as $u \in \mathcal{Z}_{(m)}^{\vee}$. If $\tilde{u}_{j k} \neq 0$, then

$$
c(\tilde{u}[k], \tilde{\lambda})= \begin{cases}n_{j k}(\lambda), & \text { if } \operatorname{wt}(\lambda)=0, j>m, \\ n_{j k}(\lambda)+1, & \text { if } \tilde{w t}^{\prime}(\lambda)=1, j>m, \\ n_{j k}(\lambda), & \text { if } \tilde{\lambda}_{i j^{\prime}}, \tilde{\lambda}_{i l} \in \mathbb{F}_{q}^{\times}, \text {with } i<j^{\prime}<j \leq m, k<l, \\ n_{j k}(\lambda), & \text { otherwise. }\end{cases}
$$

Thus,

$$
q^{\operatorname{wt}(\lambda)\left|\left\{m<j<k<\operatorname{lc}(\lambda) \mid u_{j k} \neq 0\right\}\right|} \prod_{\text {left corners } u_{j k}} q^{n_{j k}(\lambda)}=\prod_{k} q^{c(\tilde{u}[k], \tilde{\lambda})} .
$$

If $\chi^{\lambda}(u) \neq 0$, and we have no configurations of the form

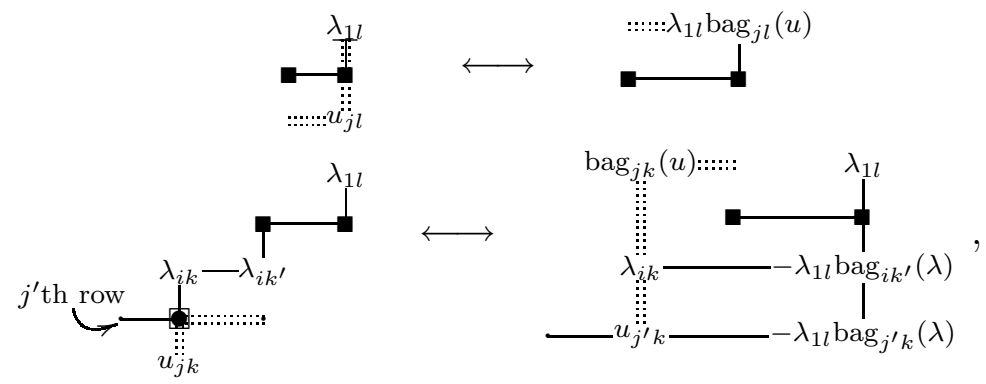


then both character formulas are equal. If (12) occurs then both the path character formula and the comb character formula get a factor of

$$
\theta\left(\lambda_{1 l} \operatorname{bag}_{j l}(u)\right) \text {. }
$$

If (13) occurs then the path character formula gets a factor

$$
\theta\left(\lambda_{j^{\prime} k} u_{j^{\prime} k}\right) \theta\left(\operatorname{bag}_{j k}(u) \operatorname{bag}_{i k}(\lambda)\right),
$$

and the comb character formula gets a factor of

$$
\theta\left(-\left(-\lambda_{1 l} \operatorname{bag}_{i k^{\prime}}(\lambda)\right)^{-1} \lambda_{i k}\left(\operatorname{bag}_{j k}(u) \lambda_{1 l}+u_{j^{\prime} k}\left(-\lambda_{1 l} \operatorname{bag}_{j^{\prime} k}(\lambda)\right)\right)\right),
$$

However, a simple computation shows that these two quantities are equal. Thus, the character formulas for the two types of representatives are equal.

\subsection{Example}

Let $n=7, m=4$,

$$
u=\left(\begin{array}{ccccccc}
1 & 0 & 0 & 0 & a & 0 & 0 \\
0 & 1 & 0 & 0 & 0 & d & 0 \\
0 & 0 & 1 & e & 0 & 0 & 0 \\
0 & 0 & 0 & 1 & b & c & 0 \\
0 & 0 & 0 & 0 & 1 & 0 & 0 \\
0 & 0 & 0 & 0 & 0 & 1 & 0 \\
0 & 0 & 0 & 0 & 0 & 0 & 1
\end{array}\right) \quad \text { and } \quad \lambda=\left(\begin{array}{ccccccc}
0 & 0 & 0 & 0 & 0 & 0 & x \\
0 & 0 & 0 & 0 & 0 & 0 & y \\
0 & 0 & 0 & 0 & 0 & 0 & 0 \\
0 & 0 & 0 & 0 & z & 0 & 0 \\
0 & 0 & 0 & 0 & 0 & 0 & 0 \\
0 & 0 & 0 & 0 & 0 & 0 & 0 \\
0 & 0 & 0 & 0 & 0 & 0 & 0
\end{array}\right),
$$

so the decorated superimposed matrix of $u-1$ and $\lambda$ is

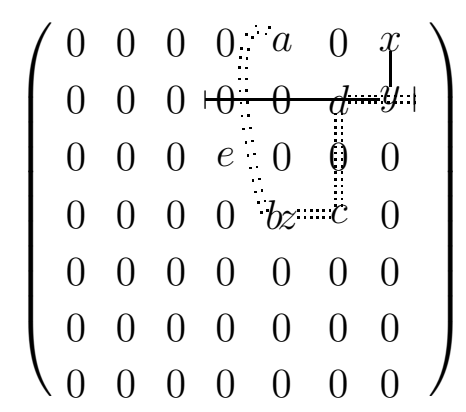

All the connected components are pairwise path compatible,

Thus,
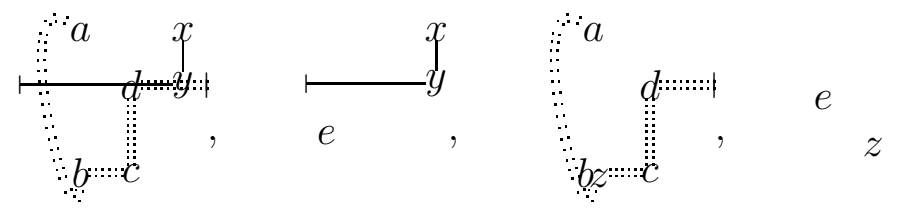

$$
\chi^{\lambda}(u) \neq 0
$$

if and only if $\operatorname{bag}(d) \operatorname{bag}(y)=1$ if and only if $d\left(-c^{-1}\right) b\left(-a^{-1}\right) y\left(-x^{-1}\right)=1$. If $\chi^{\lambda}(u) \neq 0$, then

$$
\chi^{\lambda}(u)=q^{4} \theta(b z) \frac{1}{q^{3}} \cdot 1 .
$$




\section{Interpolating from $U_{n}$ to $U_{n-1}$}

This section uses a restriction rule from $U_{(m-1)}$ to $U_{(m)}$ for supercharacters to deduce a restriction rule from $U_{n}$ to $U_{n-1}$.

\subsection{A restriction rule for $U_{(m)}$}

Note that if $\lambda \in \mathcal{Z}_{(m-1)}$, then $\lambda \in \mathcal{Z}_{(m)}$ unless $\operatorname{lc}(\lambda)=m$. Let $\lambda^{\downarrow} \in \mathcal{Z}_{(m)}$ be given by

$$
\lambda^{\downarrow}= \begin{cases}\lambda, & \text { if } \operatorname{lc}(\lambda) \neq m, \\ \lambda-\lambda_{i m} e_{i m} & \text { if } \operatorname{lc}(\lambda)=m \text { and } i \text { is minimal so } \lambda_{i m} \neq 0 .\end{cases}
$$

Then $\lambda^{\downarrow}$ is the path orbit representative of $U_{(m)} \lambda U_{(m)}$.

For $u \in \mathcal{Z}_{(m)}^{\vee}$, let

$$
u^{\uparrow}= \begin{cases}u-u_{1 j} e_{1 j}-u_{m k} e_{m k}-u_{1 j} u_{m j}^{-1} u_{m k} e_{1 k}, & \text { if } u_{1 j}, u_{m j}, u_{m k} \in \mathbb{F}_{q}^{\times}, j<k, \\ u-u_{1 j} e_{1 j}, & \text { if } u_{1 j}, u_{m j} \in \mathbb{F}_{q}^{\times}, \operatorname{wt}(u-1)=1, \\ u, & \text { otherwise, }\end{cases}
$$

so $u^{\uparrow}$ is the path orbit representative of $U_{(m-1)} u U_{(m-1)}$.

Theorem 5.1. Let $\lambda \in \mathcal{Z}_{(m-1)}$. Then

$\operatorname{Res}_{U_{(m)}}^{U_{(m-1)}}\left(\chi^{\lambda}\right)= \begin{cases}\chi^{\lambda^{\downarrow}}, & \text { if } \operatorname{lc}(\lambda)=m \text { or } \operatorname{wt}(\lambda)=0, \\ q \chi^{\lambda^{\downarrow}}, & \text { if } \operatorname{lc}(\lambda)>m, \operatorname{wt}(\lambda)=1, \lambda_{m j} \neq 0 \text { for some } j>\operatorname{lc}(\lambda), \\ \sum_{t \in \mathbb{F}_{q}} \chi^{\lambda^{\downarrow}+t e_{m \operatorname{lc}(\lambda)},}, & \text { if } \operatorname{lc}(\lambda)>m, \operatorname{wt}(\lambda)=1, \lambda_{m j}=0 \text { for all } j>\operatorname{lc}(\lambda) .\end{cases}$

Proof. Since at most one component of $\lambda$ can satisfy case three of Theorem 5.1, it suffices to prove the statement for the case that $G_{[\lambda]}$ has one connected component. Note that Case 2 does not occur unless $G_{[\lambda]}$ has at least two components, and it follows directly from cases 1 and 3 .

First, suppose that $S_{\lambda}=\emptyset$. Then $\operatorname{wt}(\lambda)=\operatorname{lc}(\lambda)=0$, and $\lambda^{\downarrow}=\lambda$. Since the support of $\lambda$ excludes the first row, the conditions implied by $\mathcal{P}_{(m)}$ are the same as the conditions implied by $\mathcal{P}_{(m-1)}$ in the character formulae and $\operatorname{Res}_{U_{(m)}}^{U_{(m-1)}}\left(\chi^{\lambda}\right)=\chi^{\lambda \downarrow}$.

Suppose that $\lambda=\left[S_{\lambda}\right]$. If $\left|S_{\lambda}\right|=1$ with $\operatorname{lc}(\lambda)=m$, then $\operatorname{br}(\lambda)=1$. In this case, $1 \not \leq m$ in $\mathcal{P}_{(m)}$ implies $\operatorname{Res}_{U_{(m)}}^{U_{(m-1)}}\left(\chi^{\lambda}\right)=\mathbb{1}_{U_{(m)}}=\chi^{\lambda \downarrow}$.

For the rest of the proof we will assume $\lambda=\left[S_{\lambda}\right]$ and $\left|S_{\lambda}\right|>1$ or $\operatorname{lc}(\lambda)>m$. Note that if $\operatorname{lc}(\lambda)>m$, wt $(\lambda)=1$, and $\lambda_{m j}=0$ for all $j>k$, then

$$
\sum_{t \in \mathbb{F}_{q}} \chi^{\lambda^{\downarrow}+t e_{m k}}(u)= \begin{cases}\chi^{\lambda^{\downarrow}}(u) \sum_{t \in \mathbb{F}_{q}} \theta\left(t u_{m, \operatorname{lc}(\lambda)}\right), & \text { if } u_{m k}=0, k<\operatorname{lc}(\lambda), \\ \chi^{\lambda^{\downarrow}}(u), & \text { otherwise. }\end{cases}
$$

Thus, all three cases of Theorem 5.1 require an understanding of the relationship between the values $\chi^{\lambda^{\downarrow}}(u)$ and $\chi^{\lambda}\left(u^{\uparrow}\right)$. 
Since $\chi^{\lambda \downarrow}$ is a supercharacter, by Lemma 4.1 WLOG we assume $G_{u-1}$ has one connected component. It follows from the combinatorics that the two values $\chi^{\lambda}\left(u^{\uparrow}\right)$ and $\chi^{\lambda^{\downarrow}}(u)$ are simultaneously zero or nonzero unless $\operatorname{wt}(\lambda)=1, u_{m, \operatorname{lc}(\lambda)} \neq 0$, and $u_{m k}=0$ for $k>\operatorname{lc}(\lambda)$. However, in this exceptional case,

$$
\sum_{t \in \mathbb{F}_{q}} \chi^{\lambda^{\downarrow}+t e_{m k}}(u)=\chi^{\lambda^{\downarrow}}(u) \sum_{t \in \mathbb{F}_{q}} \theta\left(t u_{m, \operatorname{lc}(\lambda)}\right)=0=\chi^{\lambda}\left(u^{\uparrow}\right) .
$$

Suppose $\chi^{\lambda}\left(u^{\uparrow}\right)$ and $\chi^{\lambda \downarrow}(u)$ are both nonzero. By inspection,

$$
\frac{\chi^{\lambda}(1)}{\chi^{\lambda^{\downarrow}}(1)}= \begin{cases}1, & \text { if } \operatorname{lc}(\lambda)=m \text { or } \operatorname{wt}(\lambda)=0, \\ q, & \text { otherwise. }\end{cases}
$$

Since $\lambda^{\downarrow}$ has the same bottom corners as $\lambda$ and $u^{\uparrow}$ has the same left corners as $u$,

$$
\frac{q^{\operatorname{wt}(\lambda) \#\left\{m-1<j<\operatorname{lc}(\lambda) \mid u_{m-1, j}^{\uparrow} \neq 0\right\}} \prod_{\text {left corners } u_{j k}^{\uparrow}} q^{n_{j k}(\lambda)}}{q^{\operatorname{wt}(\lambda) \#\left\{m<j<\operatorname{lc}\left(\lambda^{\downarrow}\right) \mid u_{m j} \neq 0\right\}} \prod_{\text {left corners } u_{j k}} q^{n_{j k}\left(\lambda^{\downarrow}\right)}}=\frac{q^{\operatorname{wt}(\lambda) \#\left\{m-1<j<\operatorname{lc}(\lambda) \mid u_{m-1, j}^{\uparrow} \neq 0\right\}}}{q^{\operatorname{wt}(\lambda) \#\left\{m<j<\operatorname{lc}\left(\lambda^{\downarrow}\right) \mid u_{m j} \neq 0\right\}}} .
$$

Note that

$\theta(\lambda(u-1))= \begin{cases}\theta\left(\lambda^{\downarrow}(u-1)\right) \theta\left(\lambda_{i m} u_{i m}\right), & \text { if } \operatorname{lc}(\lambda)=m, i \text { is minimal such that } \lambda_{i m} \neq 0, \\ \theta\left(\lambda^{\downarrow}(u-1)\right), & \text { otherwise. }\end{cases}$

However, in the first case, $u_{i m} \neq 0$ implies $\chi^{\lambda}(u)=0$, since $m<m+1$ and $u \in \mathcal{Z}_{(m)}$. Thus, for all $\operatorname{lc}(\lambda)$ with $\chi^{\lambda}(u) \neq 0$,

$$
\theta\left(\lambda^{\downarrow}(u-1)\right)=\theta(\lambda(u-1)) .
$$

In fact, if $S_{u-1}=\emptyset$ or $\operatorname{br}(u-1)<m$, then $u^{\uparrow}=u$ and $\theta(\lambda(u-1))=\theta\left(\lambda\left(u^{\uparrow}-1\right)\right)$. In these cases we also have $\Theta\left(u^{\uparrow}, \lambda\right)=\Theta\left(u, \lambda^{\downarrow}\right)$.

Suppose $S_{u-1} \neq \emptyset$ and $\operatorname{br}(u-1)=m$. If $S_{u-1}=\left\{u_{1 k}, u_{m k}\right\}$, then $S_{u^{\uparrow}-1}=\left\{u_{m k}\right\}$, and

$$
\theta(\lambda(u-1))=\theta\left(\lambda_{1 k} u_{1 k}\right) \theta\left(\lambda\left(u^{\uparrow}-1\right)\right) .
$$

However, since $\lambda \in \mathcal{Z}_{(m-1)}, \lambda_{1 k} \neq 0$ implies $\chi^{\lambda}\left(u^{\uparrow}\right)=\chi^{\lambda}(u)=0$.

If $\left\{u_{1 j}, u_{m j}, u_{m k}\right\} \subseteq S_{u-1}$ with $j<k$, then $u_{1 j}^{\uparrow}=u_{m k}^{\uparrow}=0, u_{m j}^{\uparrow}=u_{m j}$, and $u_{1 k}^{\uparrow}=$ $-u_{1 j} u_{m j}^{-1} u_{m k}$. Thus,

$$
\theta\left(-\lambda_{1 k} u_{1 j} u_{m j}^{-1} u_{m k}\right) \theta(\lambda(u-1))=\theta\left(\lambda_{1 j} u_{1 j}+\lambda_{m k} u_{m k}\right) \theta\left(\lambda\left(u^{\uparrow}-1\right)\right) .
$$

If $\lambda_{1 j} \neq 0$ or $\lambda_{m k} \neq$, then $\chi^{\lambda}(u)=0$, so

$$
\theta\left(-\lambda_{1 k} u_{1 j} u_{m j}^{-1} u_{m k}\right) \theta(\lambda(u-1))=\theta\left(\lambda\left(u^{\uparrow}-1\right)\right) .
$$

If $\lambda_{1 k} \neq 0$, then $\chi^{\lambda}(u) \neq 0$ only if 
(1) $S_{u-1}=\left\{u_{1 j}, u_{m j}, u_{m k}\right\}$ and $\lambda_{i k} \neq 0$ for some $1<i<k$,

(2) there exists $1<i<k$ such that $u_{i k}, \lambda_{i k} \in \mathbb{F}_{q}^{\times}$.

In both cases,

$$
\Theta(u, \lambda) \theta\left(-\lambda_{1 k} u_{1 j} u_{m j}^{-1} u_{m k}\right)=\Theta\left(u^{\uparrow}, \lambda\right) .
$$

Note that in the first case, $\theta_{E B\left(u^{\uparrow}, \lambda\right)}=\theta\left(-\lambda_{1 k} u_{1 j} u_{m j}^{-1} u_{m k}\right)$.

Thus, for all $u \in \mathcal{Z}_{(m)}$ and $\lambda \in \mathcal{Z}_{(m-1)}$ such that $\chi^{\lambda}(u) \neq 0$,

$$
\Theta\left(u, \lambda^{\downarrow}\right) \theta\left(\lambda^{\downarrow}(u-1)\right)=\Theta(u, \lambda) \theta(\lambda(u-1))=\Theta\left(u^{\uparrow}, \lambda\right) \theta\left(\lambda\left(u^{\uparrow}-1\right)\right) .
$$

We now use (16), (17), and (18) to check the cases of the theorem. Suppose $\operatorname{lc}(\lambda)=m$ or $\operatorname{wt}(\lambda)=0$, then by $(16),(17)$, and (18),

$$
\chi^{\lambda \downarrow}(u)=\chi^{\lambda}\left(u^{\uparrow}\right) .
$$

Suppose $\operatorname{lc}(\lambda)>m$ and $\operatorname{wt}(\lambda)=1$. If $\lambda_{m j}=0$ for all $j \geq \operatorname{lc}(\lambda)$, then by (14),

$$
\sum_{t \in \mathbb{F}_{q}} \chi^{\lambda \downarrow+t e_{m \mathrm{lc}(\lambda)}}(u)= \begin{cases}\chi^{\lambda \downarrow}(u) \sum_{t \in \mathbb{F}_{q}} \theta\left(t u_{m, \operatorname{lc}(\lambda)}\right), & \text { if } u_{m j}=0, j<\operatorname{lc}(\lambda), \\ \chi^{\lambda^{\downarrow}}(u), & \text { otherwise. }\end{cases}
$$

In the first case, $\chi^{\lambda}(1)=q \chi^{\lambda^{\downarrow}}(1)$ and the ratio in (17) is 1. Thus, by (18) and (15) in this case,

$$
\begin{aligned}
\sum_{t \in \mathbb{F}_{q}} \chi^{\lambda^{\downarrow}+t e_{m \operatorname{lc}(\lambda)}}(u) & = \begin{cases}q^{-1} \chi^{\lambda^{\downarrow}}\left(u^{\uparrow}\right) q, & \text { if } u_{m \operatorname{lc}(\lambda)}=0, \\
\chi^{\lambda}\left(u^{\uparrow}\right)(=0), & \text { otherwise. }\end{cases} \\
& =\chi^{\lambda}\left(u^{\uparrow}\right)
\end{aligned}
$$

In the second case of $(14), \chi^{\lambda}(1)=q \chi^{\lambda^{\downarrow}}(1)$ and the ratio in (17) is $q$. Thus,

$$
\sum_{t \in \mathbb{F}_{q}} \chi^{\lambda \downarrow+t e_{m \operatorname{lc}(\lambda)}}(u)=\chi^{\lambda \downarrow}(u)=q^{-1} \chi^{\lambda}\left(u^{\uparrow}\right) q=\chi^{\lambda}\left(u^{\uparrow}\right)
$$

completing the last case.

\subsection{A restriction rule for $U_{n}$}

For $\lambda \in \mathcal{S}_{n}(q)$, and $i, k \in \mathbb{Z}_{\geq 1}$, let

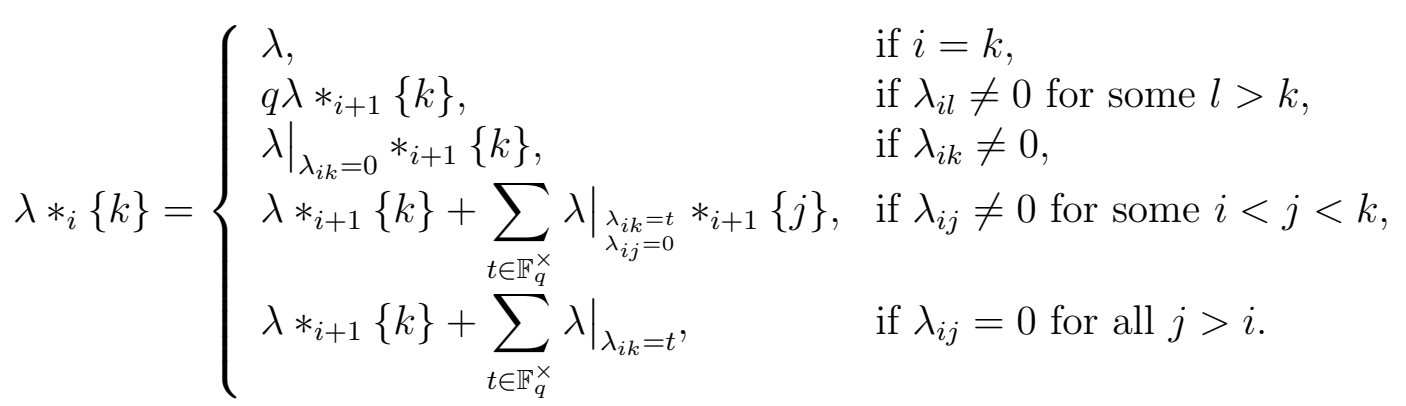


We will extend this product to supercharacters by the convention

$$
\chi^{\mu} *_{i} \chi^{\{k\}}=\sum_{\lambda} c_{\mu k}^{\lambda} \chi^{\lambda}, \quad \text { if } \quad \mu *_{i}\{k\}=\sum_{\lambda} c_{\mu k}^{\lambda} \lambda
$$

Corollary 5.1. For $\lambda \in \mathcal{S}_{n}(q)$,

$$
\operatorname{Res}_{U_{n-1}}^{U_{n}}\left(\chi^{\lambda}\right)= \begin{cases}\chi^{\lambda} *_{1} \chi^{\{k\}}, & \text { if } \lambda_{1 k} \neq 0, \text { for some } 1<k \\ \chi^{\lambda}, & \text { otherwise }\end{cases}
$$

Proof. Note that for $1 \leq m \leq n$, Theorem 5.1 implies

$$
\operatorname{Res}_{U_{(m)}}^{U_{(m-1)}}\left(\chi^{\lambda}\right)= \begin{cases}\chi^{\lambda}, & \text { if } \operatorname{lc}(\lambda) \neq m \text { and } \operatorname{wt}(\lambda)=0, \\ \chi^{\lambda-\lambda_{i m} e_{i m},} & \text { if } \operatorname{lc}(\lambda)=m, \\ q \chi^{\lambda}, & \text { if } \operatorname{lc}(\lambda)>m, \operatorname{wt}(\lambda)=1, \lambda_{m j} \neq 0 \text { for some } j>\operatorname{lc}(\lambda), \\ \sum_{t \in \mathbb{F}_{q}} \chi^{\lambda+t e_{m \operatorname{lc}(\lambda)},}, & \text { if } \operatorname{lc}(\lambda)>m, \operatorname{wt}(\lambda)=1, \lambda_{m j}=0, \text { for all } j>\operatorname{lc}(\lambda) .\end{cases}
$$

The multiplication given by (19) is an iterative version of the restrictions from $U_{(m-1)}$ to $U_{(m)}$, where the last two cases in (19) correspond to the last case in the restriction, depending on whether there exists $\lambda_{m j} \in \mathbb{F}_{q}^{\times}$for some $j<\operatorname{lc}(\lambda)$.

Remark. It follows that the coefficients of the restriction are polynomial in $q$.

\subsection{Examples}

Example 1. Consider the case $q=2$. Then we may choose our representatives $\lambda \in \mathfrak{n}_{n}^{*}$ and $u \in U_{n}$ to correspond to set partitions of $\{1,2, \ldots, n\}$. For example, if

$$
\lambda=\{1 \frown 5|2 \frown 6| 3 \frown 4\},
$$

then

$$
\begin{aligned}
\operatorname{Res}_{U_{5}}^{U_{6}}\left(\chi^{\lambda}\right) & =\chi^{\{1-5|2-6| 3-4\}} *_{1}\{5\} \\
& =\chi^{\{1|5| 2-6 \mid 3-4\}} *_{2}\{5\} \\
& =2 \chi^{\{1|5| 2-6 \mid 3-4\}} *_{3}\{5\} \\
& =2 \chi^{\{1|5| 2-6 \mid 3-4\}} *_{4}\{5\}+2 \chi^{\{1|2-6| 3-5 \mid 4\}} *_{4}\{4\} \\
& =2 \chi^{\{1|5| 2-6 \mid 3-4\}} *_{5}\{5\}+2 \chi^{\{1|2-6| 3-4-5\}} *_{5}\{5\}+2 \chi^{\{1|2-6| 3-5 \mid 4\}} \\
& =2 \chi^{\{1|5| 2-6 \mid 3-4\}}+2 \chi^{\{1|2-6| 3-4-5\}}+2 \chi^{\{1|2-6| 3-5 \mid 4\}}
\end{aligned}
$$

Note that the final result gives the representative of $U_{n-1}$ as a submatrix of $U_{n}$ (since by construction the first row will always be zero). To obtain a set partition of $n-1$, we would remove 1 and renumber the rest of the entries $j \mapsto j-1$. 
Alternatively, this algorithm may be viewed as a "bumping algorithm", where we replace the 1 by all other "possibilities," suitably defined.

Example 2. Linear characters of $U_{n}$ are those characters whose superclass representative satisfies, $i$ and $j$ are in the same part only if $i+1, i+2, \ldots, j-1$ are also in the part. In this case,

$$
\chi^{\lambda} *_{1}\{j\}=\chi^{\lambda}
$$

Example 3. On the opposite extreme with have the case

$$
\lambda=\{1 \frown n|2| 3|\cdots| n-1\} .
$$

In this case,

$$
\chi^{\lambda} *_{1}\{n\}=\sum_{\substack{\mu \in \mathcal{S}_{n}(q), \lambda_{i j}=0,1 \leq i<j<n}} \chi^{\mu}
$$

\subsection{An alternate embedding of $U_{n-1}$}

The paper [13] uses a different embedding of $U_{n-1}$ into $U_{n}$ (obtained by removing the last column rather than the first row). This alternate embedding gives a different restriction rule. For $\mu \in \mathcal{S}_{n}(q)$ and $j, l \in \mathbb{Z}_{\geq 1}$, let

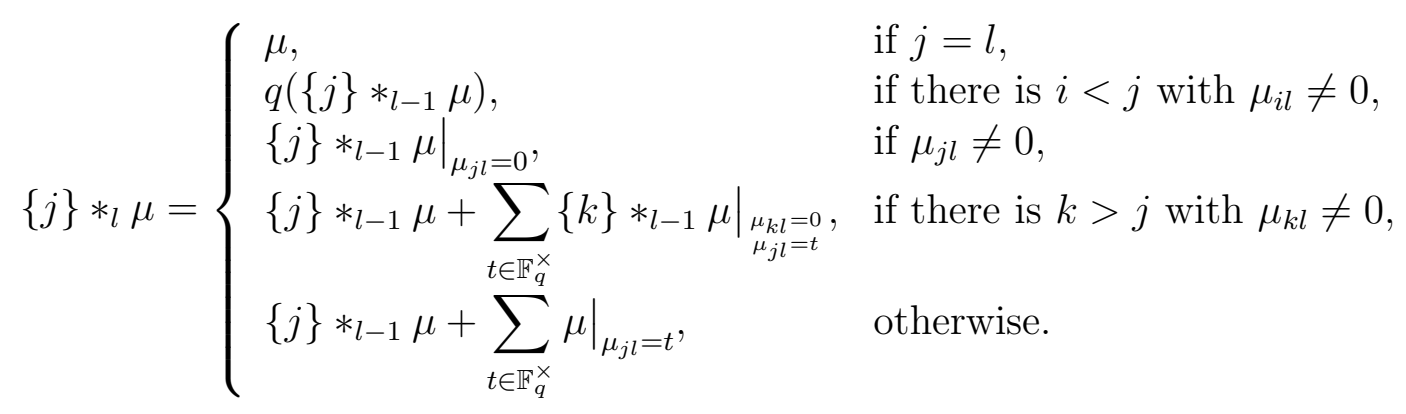

Then by symmetry arguments from [13], we obtain the following corollary for this alternate embedding of $U_{n-1}$ into $U_{n}$.

Corollary 5.2. For $\lambda \in \mathcal{S}_{n}(q)$,

$$
\operatorname{Res}_{U_{n-1}}^{U_{n}}\left(\chi^{\lambda}\right)= \begin{cases}\chi^{\{j\}} *_{n} \chi^{\lambda}, & \text { if } \lambda_{j n} \neq 0, \text { for some } j<n \\ \chi^{\lambda}, & \text { otherwise. }\end{cases}
$$

In particular, unlike in the symmetric group representation theory, the decomposition of restricted characters depends on the embedding of $U_{n-1}$ into $U_{n}$. Other than the "antitranspose" symmetry of this section, it is currently unknown what kind of combinatorial relationship might exist between the restriction coefficients of different embeddings.

Remark. The paper [13] studies the problem of induction from $U_{n-1}$ to $U_{n}$. While this is done directly, one could obtain analogous results from this paper by Frobenius reciprocity. 


\section{References}

[1] André, C. "Basic characters of the unitriangular group," J. algebra 175 (1995), 287319.

[2] André, C. "Irreducible characters of finite algebra groups," Matrices and group representations Coimbra, 1998 Textos Mat. Sér B 19 (1999), 65-80.

[3] André, C. "The basic character table of the unitriangular group," J. algebra 241 (2001), 437-471.

[4] André, C. "Basic characters of the unitriangular group (for arbitrary primes)," Proc. Amer. Math. Soc. 130 (2002), 1934-1954.

[5] André, C; Neto, A. "Super-characters of finite unipotent groups of types $B_{n}, C_{n}$ and $D_{n}, "$ August 2006 preprint.

[6] André, C; Nicolás, A. "Supercharacters of the adjoint group of a finite radical ring," August 2006 preprint.

[7] Arregi, J; Vera-Lopez, A. "Computing in unitriangular matrices over finite fields." Linear algebra and Appl. 387 (2004), 193-219.

[8] Arias-Castro E; Diaconis, P; Stanley, R. "A super-class walk on upper-triangular matrices," J. algebra 278 (2004), 739-765.

[9] Boyarchenko, M; Drinfeld, V. "A motivated introduction to character sheaves and the orbit method for unipotent groups in positive chracteristic." Preprint (2006), arXiv.0609769.

[10] Diaconis, P; Isaacs, M. "Supercharacters and superclasses for algebra groups," To appear in Trans. Amer. Math. Soc., 2006.

[11] Diaconis, P; Saloff-Coste, L. "Comparison techniques for random walk on finite groups," Ann. Probab. 21 (1993), 2131-2156.

[12] Diaconis, P; Thiem, N. "Supercharacter formulas for pattern groups." To appear in Trans. Amer. Math. Soc., 2007.

[13] Marberg, E; Thiem, N. "Superinduction for pattern groups." A 2007 preprint.

[14] Yan, N. Representation theory of the finite unipotent linear groups, Unpublished Ph.D. Thesis, Department of mathematics, University of Pennsylvania, 2001.

[15] Yan, N. "Representations of finite unipotent linear groups by the method of clusters," 2006 preprint. 